\title{
Contributions to the theory of $F$-automatic sets
}

\author{
Christopher Hawthorne*
}

November 26, 2020

\begin{abstract}
Fix an abelian group $\Gamma$ and an injective endomorphism $F: \Gamma \rightarrow \Gamma$. Improving on the results of [2], new characterizations are here obtained for the existence of spanning sets, $F$-automaticity, and $F$-sparsity. The model theoretic status of these sets is also investigated, culminating with a combinatorial description of the $F$-sparse sets that are stable in $(\Gamma,+)$, and a proof that the expansion of $(\Gamma,+)$ by any $F$-sparse set is NIP. These methods are also used to show for prime $p \geq 7$ that the expansion of $\left(\mathbb{F}_{p}[t],+\right)$ by multiplication restricted to $t^{\mathbb{N}}$ is NIP.
\end{abstract}

\section{Contents}

1 Introduction 1

2 Preliminaries 2

3 Existence of spanning sets 4

4 A characterization of F-automaticity 8

5 A characterization of $F$-sparsity 10

$6 \quad F$-sparse sets and stable expansions of finitely generated abelian groups 15

7 NIP expansions of $(\Gamma,+) \quad 18$

\section{Introduction}

A set $S$ of natural numbers is said to be $p$-automatic if the set of representations base $p$ of the elements of $S$ forms a regular language - meaning that it is recognized by a finite automaton. Motivated by the isotrivial Mordell-Lang problem, Bell and Moosa were led in [2] to extend this notion to the context where the natural numbers are replaced by an abelian group $\Gamma$ and multiplication by $p$ is replaced by a fixed injective endomorphism $F$ of $\Gamma$. The intended examples were when $\Gamma$ is a finitely generated subgroup of a semiabelian variety $G$ over a finite field $\mathbb{F}_{q}$, with $\Gamma$ preserved by the $q$-power Frobenius endomorphism $F$ of $G$. In that setting, Bell and Moosa show in [2] that if $X$ is a closed subvariety of $G$ then $X \cap \Gamma$ is $F$-automatic; they do so using the isotrivial Mordell-Lang theorem of Moosa and Scanlon in [6], which describes $X \cap \Gamma$. These results have recently been made effective and generalized to arbitrary commutative algebraic groups by Bell, Ghioca, and Moosa in [1].

Our interest here is in the general theory of $F$-automata. The precise definition of an $F$-automatic subset of $\Gamma$ is recalled in Section 2, but let us give an informal explanation now. First one needs to know that $(\Gamma, F)$ admits a "spanning set": this is a finite subset $\Sigma$ such that every element of $\Gamma$ can be expressed as $s_{0}+F s_{1}+\cdots+F^{n} s_{n}$ for some $s_{0}, \ldots, s_{n} \in \Sigma$. Then a subset $S$ of $\Gamma$ is said to be $F$-automatic if the set of words $s_{0} s_{1} \cdots s_{n}$ such that $s_{0}+F s_{1}+\cdots+F^{n} s_{n} \in S$ forms a regular language on the alphabet $\Sigma$.

\footnotetext{
${ }^{*}$ This work was partially supported by an NSERC PGS-D and an NSERC CGS-D.
} 
Our goals in this paper are twofold: (1) to clarify and further develop the foundations of this theory, and (2) to investigate the model-theoretic properties of $F$-automatic sets, in particular with respect to the stability-theoretic hierarchy.

Regarding foundations, our main results are:

- A characterization of when $(\Gamma, F)$ admits a spanning set in terms of the existence of a height function on $\Gamma$ (Theorem 3.9) and, in the finitely generated case, in terms of the eigenvalues of $F \otimes_{\mathbb{Z}} \operatorname{id}_{\mathbb{C}}$ (Theorem 3.11).

- A characterization of $F$-automaticity in terms of kernels; this is Theorem 4.2 below. This result brings the basic theory of $F$-automatic sets closer to the classical case of $p$-automaticity.

- A characterization of $F$-sparsity. In the theory of regular languages there is a natural sparse/nonsparse dichotomy in terms of the growth of the number of accepted words of bounded length. This was extended and investigated in [2]; see Definition 5.1 below for details. We clarify some properties of $F$-sparse sets (answering a question in [2] along the way) and characterize them in terms of length functions (Theorem 5.9). As a consequence, we obtain in Corollary 5.11 a useful criterion for verifying $F$-sparsity; this criterion is used by Bell-Ghioca-Moosa in the recent preprint [1] on effective isotrivial Mordell-Lang.

We next turn to the model-theoretic analysis. Here our main results are:

- An explicit characterization of stable $F$-sparse sets as finite Boolean combinations of translates of finite sums of sets of the form $\left\{a+F a+\cdots+F^{n} a: n<\omega\right\}$-these are the "groupless $F$-sets" of [6]. That the groupless $F$-sets are stable is from [6]; the converse is Theorem 6.3 below.

- The production of some new NIP expansions of $(\Gamma,+)$; see Theorem 7.7. This includes $(\Gamma,+, A)$ for any $F$-sparse $A \subseteq \Gamma$, but it also includes some examples that are not even $F$-automatic, most notably the expansion of $\left(\mathbb{F}_{p}[t],+\right)$ by the graph of multiplication restricted to $t^{\mathbb{N}}$, when $p \geq 7$. This last example is Theorem 7.8 below.

My thanks to Luke Franceschini for helping me work through the details of Example 3.1. Warm thanks to my advisor, Rahim Moosa, for his excellent guidance, thorough editing, and many helpful discussions.

\section{Preliminaries}

We first recall the relevant theory of regular languages; the interested reader is directed to [7] for further details. Fix a finite set $\Lambda$, which we will use as an alphabet. We use $\Lambda^{*}$ to denote the set of strings of elements of $\Lambda$. A language is any subset $L \subseteq \Lambda^{*}$. We use $\varepsilon$ to denote the empty string. Given $\sigma \in \Lambda^{*},|\sigma|$ denotes the length of $\sigma$.

Definition 2.1. The set of regular languages over $\Lambda$ is the smallest set of languages that contains the finite languages and is such that if $L_{1}, L_{2} \subseteq \Lambda^{*}$ are regular then so are $L_{1} \cup L_{2}, L_{1} L_{2}=\left\{\sigma \tau: \sigma \in L_{1}, \tau \in L_{2}\right\}$, and $L_{1}^{*}=\left\{\sigma_{1} \cdots \sigma_{n}: \sigma_{1}, \ldots, \sigma_{n} \in L_{1}\right\}$.

Regular languages can be characterized as those recognized by machines of a certain form:

Definition 2.2. A non-deterministic finite automaton (or $N F A$ ) is a 5 -tuple $M=\left(\Lambda, Q, q_{0}, \Omega, \delta\right.$ ), where:

- $\Lambda$ is a finite alphabet.

- $Q$ is a finite set of states.

- $q_{0} \in Q$ is the initial state.

- $\Omega \subseteq Q$ is the set of finish states.

- $\delta: Q \times \Lambda \rightarrow 2^{Q}$ is the transition function: given $(q, a) \in Q \times \Lambda$ it outputs the set of states the machine could transition to if it is in state $q$ and reads input $a$. 
We identify $\delta$ with its natural extension $Q \times \Lambda^{*} \rightarrow 2^{Q}$ given by $\delta(q, \sigma a)=\bigcup\left\{\delta\left(q^{\prime}, a\right): q^{\prime} \in \delta(q, \sigma)\right\}$. If $\sigma \in \Lambda^{*}$ we say $M$ accepts $\sigma$ if $\delta\left(q_{0}, \sigma\right) \cap \Omega \neq \emptyset$; the set of such $\sigma$ is the language recognized by $M$. If $|\delta(q, a)|=1$ for all $q \in Q$ and $a \in \Lambda$ we say $M$ is a deterministic finite automaton (or DFA).

Fact 2.3 ([7, Lemma 2.2, Sections 3.2 and 3.3]). If $L \subseteq \Lambda^{*}$ then the following are equivalent:

- $L$ is regular.

- $L$ is recognized by some DFA.

- $L$ is recognized by some NFA.

A note on exponential notation: we use $\Lambda^{r}$ to denote the alphabet of $r$-tuples of elements of $\Lambda$, and we use $\Lambda^{(r)}$ to denote the language $\left\{\sigma \in \Lambda^{*}:|\sigma|=r\right\}$.

Throughout the paper, we fix an infinite abelian group $\Gamma$ equipped with some injective endomorphism $F: \Gamma \rightarrow \Gamma$. We let $\mathbb{Z}[F]$ denote the subring of $\operatorname{End}(\Gamma)$ generated by $F$, and consider $\Gamma$ as a $\mathbb{Z}[F]$-module. Our context is slightly more general than that of [2]: they require that $\Gamma$ be a finitely generated abelian group, which we do not. Most of the results of [2] go through in this context with no additional effort.

Following [2], given a string $s_{0} \cdots s_{n}$ of elements in $\Gamma$ we let

$$
\left[s_{0} \cdots s_{n}\right]_{F}=s_{0}+F s_{1}+\cdots+F^{n} s_{n}
$$

Note that when $(\Gamma, F)=(\mathbb{Z}, d)$ and $s_{i} \in\{0, \ldots, d-1\}$ this is just computing the number represented by $s_{0} \cdots s_{n}$ base $d$. Given a set $L$ of strings of elements of $\Gamma$, we let

$$
[L]_{F}=\left\{[\sigma]_{F}: \sigma \in L\right\} .
$$

Definition 2.4. If $\Sigma$ is a finite subset of $\Gamma$ we say $\Sigma$ is an $F$-spanning set (for $\Gamma$ ) if it satisfies the following axioms:

(i) For all $a \in \Gamma$ there is $\sigma \in \Sigma^{*}$ such that $a=[\sigma]_{F}$.

(ii) $0 \in \Sigma$, and if $a \in \Sigma$ then $-a \in \Sigma$.

(iii) If $a_{1}, \ldots, a_{5} \in \Sigma$ then $a_{1}+\cdots+a_{5} \in \Sigma+F \Sigma$.

(iv) If $a_{1}, a_{2}, a_{3} \in \Sigma$ and $a_{1}+a_{2}+a_{3} \in F \Gamma$ then $a_{1}+a_{2}+a_{3} \in F \Sigma$.

Conditions (ii-iv) are included largely for bookkeeping reasons; the main condition is (i). It says that every element of $\Gamma$ has an " $F$-expansion with digits in $\Sigma$ ". Note that the existence of an $F$-spanning set will imply that $\Gamma$ is finitely generated as a $\mathbb{Z}[F]$-module, and that $\Gamma / F \Gamma$ is finite.

It is pointed out in $\left[2\right.$, Lemmas 5.6 and 5.7] that for $r>0$ if $\Sigma$ is an $F$-spanning set then $\left[\Sigma^{(r)}\right]_{F}$ is both an $F$-spanning set and an $F^{r}$-spanning set. ${ }^{1}$

Definition 2.5. We say $A \subseteq \Gamma$ is $F$-automatic if there is an $F^{r}$-spanning set $\Sigma$ for some $r>0$ such that $\left\{\sigma \in \Sigma^{*}:[\sigma]_{F^{r}} \in A\right\}$ is regular.

The following is a useful strengthening of [2, Proposition 6.3]:

Proposition 2.6. Suppose $A \subseteq \Gamma$ is F-automatic. Then for any $r>0$ and any $F^{r}$-spanning set $\Sigma$, $\left\{\sigma \in \Sigma^{*}:[\sigma]_{F^{r}} \in A\right\}$ is regular.

Proof. By $F$-automaticity there is $r_{0}>0$ and an $F^{r_{0}}$-spanning set $\Sigma_{0}$ such that $\left\{\sigma \in \Sigma_{0}^{*}:[\sigma]_{F^{r_{0}}} \in A\right\}$ is regular. Now $\Theta:=\left[\Sigma^{\left(r_{0}\right)}\right]_{F^{r}}$ is an $F^{r r_{0}}$-spanning set, and by [2, Proposition 6.3] we have that $\left\{\sigma \in \Theta^{*}\right.$ : $\left.[\sigma]_{F^{r r_{0}}} \in A\right\}$ is regular. Suppose it is recognized by the automaton $M=\left(\Theta, Q, q_{0}, \Omega, \delta\right)$. Now define a new automaton $M^{\prime}=\left(\Sigma, Q \times \Sigma^{\left(<r_{0}\right)},\left(q_{0}, \varepsilon\right), \Omega^{\prime}, \delta^{\prime}\right)$ by

$$
\begin{aligned}
\delta^{\prime}((q, \sigma), a) & = \begin{cases}\left(\delta\left(q,[\sigma a]_{F^{r}}\right), \varepsilon\right) & \text { if }|\sigma|=r_{0}-1 \\
(q, \sigma a) & \text { if }|\sigma|<r_{0}-1\end{cases} \\
\Omega^{\prime} & =\left\{(q, \sigma): \delta\left(q,[\sigma]_{F^{r}}\right) \in \Omega\right\}
\end{aligned}
$$

\footnotetext{
${ }^{1}$ What we denote $\left[\Sigma^{(r)}\right]_{F}$ is denoted simply $\Sigma^{(r)}$ in [2]; recall that for us $\Sigma^{(r)}$ denotes the set of strings over $\Sigma$ of length $r$.
} 
(Note if $|\sigma|<r_{0}$ that $[\sigma]_{F^{r}}=\left[\sigma 0^{r_{0}-|\sigma|}\right]_{F^{r}} \in \Theta$, so $\delta\left(q,[\sigma]_{F^{r}}\right)$ is defined.) Given $\sigma \in \Sigma^{*}$ we can write $\sigma=\sigma_{1} \cdots \sigma_{n+1}$ where $\left|\sigma_{1}\right|=\cdots=\left|\sigma_{n}\right|=r_{0}$ and $\left|\sigma_{n+1}\right|<r_{0}$. Now, $M^{\prime}$ accepts $\sigma$ if and only if $M$ accepts $\left[\sigma_{1}\right]_{F^{r}} \cdots\left[\sigma_{n+1}\right]_{F^{r}}$ (where again $\left[\sigma_{n+1}\right]_{F^{r}}=\left[\sigma_{n+1} 0^{r_{0}-\left|\sigma_{n+1}\right|}\right]_{F^{r}} \in \Theta$ ), which is in turn equivalent to $[\sigma]_{F^{r}}=$ $\left[\left[\sigma_{1}\right]_{F^{r}} \cdots\left[\sigma_{n+1}\right]_{F^{r}}\right]_{F^{r r_{0}}} \in A$. So $M^{\prime}$ recognizes $\left\{\sigma \in \Sigma^{*}:[\sigma]_{F^{r}} \in A\right\}$, as desired. $\square$ Proposition 2.6

\section{$3 \quad$ Existence of spanning sets}

We first study what it means for $\Gamma$ to admit an $F$-spanning set. It is clear in [2] that the authors do not expect all finitely generated abelian groups to admit spanning sets, but no example was given. Here is one:

Example 3.1. Consider $\Gamma=\mathbb{Z}^{2}$. Fix $T \in M_{2}(\mathbb{Z})$ invertible and diagonalizable over $\mathbb{C}$, and with an eigenvalue $\mu$ with $|\mu|<1$; for example $T=\left(\begin{array}{ll}1 & 1 \\ 1 & 0\end{array}\right)$. Let $F: \mathbb{Z}^{2} \rightarrow \mathbb{Z}^{2}$ be the associated linear map on $\mathbb{Z}^{2}$. Suppose for contradiction that there were an $F$-spanning set $\Sigma$ for $\mathbb{Z}^{2}$. Let $\{v, w\} \subseteq \mathbb{C}^{2}$ be an eigenbasis for $T$, say with $T v=\mu v$ and $T w=\nu w$. Write each element of $\Sigma$ (uniquely) in the form $a v+b w$ for $a, b \in \mathbb{C}$, and let $M$ be the largest $|a|$ obtained in this way. Given $x \in \mathbb{Z}^{2}$ we can write

$$
x=\left[s_{0} \cdots s_{n}\right]_{F}=\sum_{i=0}^{n} T^{i}\left(a_{i} v+b_{i} w\right)=\left(\sum_{i=0}^{n} a_{i} \mu^{i}\right) v+\left(\sum_{i=0}^{n} b_{i} \nu^{i}\right) w
$$

where $s_{i}=a_{i} v+b_{i} w \in \Sigma$. So by independence of $\{v, w\}$ if we write $x=a v+b w$ then

$$
|a|=\left|\sum_{i=0}^{n} a_{i} \mu^{i}\right| \leq \sum_{i=0}^{n}\left|a_{i}\right||\mu|^{i} \leq M \sum_{i=0}^{\infty}|\mu|^{i} \leq M(1-|\mu|)^{-1}
$$

On the other hand, if we had $a \neq 0$ then since $\mathbb{Z}^{2}$ is closed under integer scaling there would be some $x \in \mathbb{Z}^{2}$ such that the corresponding $a$ did not satisfy $|a| \leq M(1-|\mu|)^{-1}$, a contradiction. So $a=0$, and $\mathbb{Z}^{2} \subseteq \mathbb{C} w$, contradicting the fact that the $\mathbb{C}$-linear span of $\mathbb{Z}^{2}$ is all of $\mathbb{C}^{2}$. So no $F$-spanning set exists for $\mathbb{Z}^{2}$.

Bell and Moosa [2] give a sufficient condition for $\Gamma$ to admit an $F^{r}$-spanning set for some $r$ (under the assumption that $\Gamma / F \Gamma$ is finite) in terms of the existence of what they call a height function on $\Gamma$. Our first goal is to show that this is also necessary.

Definition 3.2. A height function for $(\Gamma, F)$ is a map $h: \Gamma \rightarrow \mathbb{R}_{\geq 0}$ satisfying the following:

(Symmetry and triangle inequality) There are $\alpha, \kappa \in \mathbb{R}$ with $D>1$ and $\kappa>0$ such that $h(-a) \leq \alpha D h(a)+\kappa$ and $h(a+b) \leq \alpha(h(a)+h(b))+\kappa$ for all $a, b \in \Gamma$.

(Northcott property) For all $N \in \mathbb{N}$ there are finitely many $a \in \Gamma$ with $h(a) \leq N$.

(Canonicity) There is $\beta \in \mathbb{R}$ with $\beta>1$ such that $h(F a)>\beta h(a)$ for cofinitely many $a \in \Gamma$.

We find it more useful to work with a variant of height that we will call "length".

Definition 3.3. A length function for $(\Gamma, F)$ is a map $\lambda: \Gamma \rightarrow \mathbb{R}_{\geq 0}$ satisfying the following:

(Symmetry) $\lambda(a)=\lambda(-a)$ for all $a \in \Gamma$.

(Ultrametric inequality) There is $D \in \mathbb{R}$ with $D \geq 1$ such that $\lambda(a+b) \leq D \max (\lambda(a), \lambda(b))$ for all $a, b \in \Gamma$.

(Northcott property) For all $N \in \mathbb{N}$ there are finitely many $a \in \Gamma$ with $\lambda(a) \leq N$.

(Canonicity) There is a finite exceptional set $A$ and $C, E \in \mathbb{R}$ with $C>1$ and $E \geq 1$ such that

- $\lambda(F a) \leq C \lambda(a)$ for all $a \in \Gamma$, and

- $\lambda\left(F^{n} a\right) \geq \frac{C^{n}}{E} \lambda(a)$ for all $a \in \Gamma \backslash A$ and $n \in \mathbb{N}$. 
Note that if $\lambda$ satisfies all the axioms of being a length function besides symmetry, then $\lambda^{\prime}(a):=$ $\max (\lambda(a), \lambda(-a))$ will be a length function.

Length and height functions are closely related. Suppose $\lambda$ is a length function for $(\Gamma, F)$ with exceptional set $A$ and associated constants $C, D, E$. Pick $r$ so that $C^{r}>E$. It is then straightforward to check that $\lambda$ is a height function for $\left(\Gamma, F^{r}\right)$ with exceptional set $A$ and associated constants $\alpha=D, \beta=C^{r} E^{-1}$, and $\kappa=0$. It is harder to derive a length function from a height function, as the axioms of height functions place no upper bound on the height of $F a$ in terms of the height of $a$. However, the height functions that arise in the isotrivial Mordell-Lang context of [2] turn out to also be length functions.

Remark 3.4. It will sometimes be convenient to assume that $C$ is large compared to some function of $D$ and $E$ (and possibly other constants). If we are willing to pass from $F$ to a power thereof, this can always be assumed: if $\lambda$ is a length function for $(\Gamma, F)$ with associated constants $C, D, E$, we can take $r$ such that $C^{r}$ satisfied the desired inequalities. Then $\lambda$ is a length function for $\left(\Gamma, F^{r}\right)$ with associated constants $C^{r}, D, E$.

Lemma 3.5. Suppose $\lambda$ is a length function for $(\Gamma, F)$ with exceptional set $A$ and associated constants $C, D, E$. By increasing $E$ we can assume that every element of the exceptional set $A$ has finite $F$-orbit.

Proof. Suppose the $F$-orbit of $a \in A$ is infinite; so there is $i_{a}$ such that $F^{i_{a}} a \notin A$. Note that all $\lambda\left(F^{i} a\right) \neq 0$ : otherwise the ultrametric inequality would imply $\lambda\left(k F^{i} a\right)=0$ for all $k$, contradicting the Northcott property. Now, for all $n \geq i_{a}$ we have

$$
\lambda\left(F^{n} a\right)=\lambda\left(F^{n-i_{a}} F^{i_{a}} a\right) \geq \frac{C^{n-i_{a}}}{E} \lambda\left(F^{i_{a}} a\right) \geq C^{n} \lambda(a)(\underbrace{C^{i_{a}} E \frac{\lambda(a)}{\lambda\left(F^{i_{a}} a\right)}}_{*})^{-1}
$$

and for $n<i_{a}$ we have

$$
\lambda\left(F^{n} a\right)=C^{n} \lambda(a)(\underbrace{C^{n} \frac{\lambda(a)}{\lambda\left(F^{n} a\right)}}_{*})^{-1}
$$

Hence taking $E^{\prime}$ to be the maximum of $E$ and all the quantities marked $*$, we get that $\lambda\left(F^{n} a\right) \geq \frac{C^{n}}{E^{\prime}} \lambda(a)$ so that $a$ is no longer exceptional with respect to this new $E^{\prime}$. Iterating this procedure for each $a \in A$ with infinite $F$-orbit, we eventually produce a new $\widetilde{E} \geq E$ such that only the elements of $A$ of finite $F$-orbit remain exceptional.

$\square$ Lemma 3.5

Remark 3.6. If there is a length function for $(\Gamma, F)$ and $a \in \Gamma$ has finite $F$-orbit, then $a$ must have finite order. Indeed, the $F$-orbit of $k a$ is finite for all $k \in \mathbb{N}$; but canonicity then implies that all $k a$ lie in the finite exceptional set.

Combining Lemma 3.5 and Remark 3.6 we may assume canonicity applies to all elements of infinite order. Here is the motivating example of a length function.

Definition 3.7. If $\Sigma$ is an $F$-spanning set for $\Gamma$, we define $\lambda_{\Sigma}: \Gamma \rightarrow \mathbb{R}_{\geq 0}$ by $\lambda_{\Sigma}(a)=2^{\ell}$, where $\ell$ is the length of the shortest $\sigma \in \Sigma^{*}$ such that $[\sigma]_{F}=a$.

Proposition 3.8. If $\Sigma$ is an $F$-spanning set for $\Gamma$ then $\lambda_{\Sigma}$ is a length function for $(\Gamma, F)$ with associated constants $C=D=E=2$ and exceptional set $\Sigma$.

Proof. We verify the axioms. Symmetry of $\lambda_{\Sigma}$ is simply by symmetry of $\Sigma$. Lemma 5.3 of [2] implies that the ultrametric inequality holds of $\lambda_{\Sigma}$ with $D=2$. Since $\Sigma$ is finite there are finitely many $\sigma \in \Sigma^{*}$ of length $\leq N$, and hence $\lambda_{\Sigma}$ satisfies the Northcott property. Finally, we prove canonicity for $\lambda_{\Sigma}$ with $C=E=2$ and exceptional set $\Sigma$. For the upper bound, note that if $a=\left[s_{0} \cdots s_{\ell}\right]_{F}$ with $s_{0}, \ldots, s_{\ell} \in \Sigma$ then $F a=\left[0 s_{0} \cdots s_{\ell}\right]_{F} ;$ so $\lambda_{\Sigma}(F a) \leq 2 \lambda_{\Sigma}(a)$. It remains to show the lower bound.

Suppose $a \notin \Sigma$. Write $\lambda_{\Sigma}(a)=2^{\ell}$ for some $\ell>1$; say $a=\left[s_{0} \cdots s_{\ell-1}\right]_{F}$. Suppose for contradiction that $\lambda_{\Sigma}\left(F^{m} a\right)<2^{m+\ell-1}$; so we can write $F^{m} a=\left[t_{0} \cdots t_{m+\ell-3}\right]_{F}$. Then

$$
F^{m} s_{0}+\cdots+F^{m+\ell-1} s_{\ell-1}=F^{m} a=t_{0}+\cdots+F^{m+\ell-3} t_{m+\ell-3}
$$


Then $t_{0} \in F \Gamma$, so by axiom (iv) we get that $t_{0}=F t_{0}^{\prime}$ for some $t_{0}^{\prime} \in \Sigma$. Inductively suppose for some $i<m-1$ we can write $t_{0}+\cdots+F^{i} t_{i}=F^{i+1} t_{i}^{\prime}$ for some $t_{i}^{\prime} \in \Sigma$. Then $F^{i+1}\left(t_{i}^{\prime}+t_{i+1}\right)=t_{0}+\cdots+F^{i+1} t_{i+1}$ which can be seen to be in $F^{i+2} \Gamma$ using Eq. (1) and the fact that $m \geq i+2$. Hence $t_{i}^{\prime}+t_{i+1} \in F \Gamma$ by injectivity of $F$, and again by axiom (iv) we can write $t_{i}^{\prime}+t_{i+1}=F t_{i+1}^{\prime}$ for some $t_{i+1} \in \Sigma$. So $t_{0}+\cdots+F^{i+1} t_{i+1}=F^{i+2} t_{i+1}^{\prime}$. It follows that $t_{0}+\cdots+F^{m-1} t_{m-1}=F^{m} t_{m-1}^{\prime}$ for some $t_{m-1}^{\prime} \in \Sigma$. (Note $t_{m-1}$ is defined since $\ell \geq 2$.) So $F^{m} a=F^{m} t_{m-1}^{\prime}+F^{m} t_{m}+\cdots+F^{m+\ell-3} t_{m+\ell-3}$, and thus

$$
a=t_{m-1}^{\prime}+\left(t_{m}+\cdots+F^{\ell-3} t_{m+\ell-3}\right)
$$

Hence by [2, Lemma 5.3] we get that $a$ can be represented by a string of length $\leq \ell-1$, contradicting our assumption that $\lambda_{\Sigma}(a)=2^{\ell}$.

$\square$ Proposition 3.8

Putting all this together, we deduce the following characterization for the existence of spanning sets, which in particular provides a converse to [2, Proposition 5.8].

Theorem 3.9. Suppose $\Gamma / F \Gamma$ is finite. The following are equivalent:

1. $\Gamma$ admits an $F^{r}$-spanning set for some $r>0$.

2. There is a length function for some $\left(\Gamma, F^{r}\right)$.

3. There is a height function for some $\left(\Gamma, F^{r}\right)$.

Proof. (1) $\Longrightarrow(2)$ is the previous proposition, and we noted $(2) \Longrightarrow(3)$ after the definition of length functions. For $(3) \Longrightarrow(1)$, we appeal to [2, Proposition 5.8]. Formally they require that $\Gamma$ be finitely generated as a group, not merely as a $\mathbb{Z}[F]$-module, but this is only used to deduce that $\Gamma / F^{r} \Gamma$ is finite for all $r$; we show that we can assume as much.

Let $S \subseteq \Gamma$ contain a representative of each coset of $F \Gamma$; we claim that $\left[S^{(r)}\right]_{F}=\left\{s_{0}+\cdots+F^{r-1} s_{r-1}\right.$ : $\left.s_{i} \in S\right\}$ contains a representative of each coset of $F^{r} \Gamma$. Indeed, given $a \in \Gamma$ we can find $s_{0} \in S$ such that $a \equiv s_{0}(\bmod F \Gamma)$. Then inductively we can find $s_{1}, \ldots, s_{r-1} \in S$ such that $F^{-1}\left(a-s_{0}\right) \equiv s_{1}+\cdots+F^{r-2} s_{r-1}$ $\left(\bmod F^{r-1} \Gamma\right)$, at which point it follows that $a \equiv s_{0}+\cdots+F^{r-1} s_{r-1}\left(\bmod F^{r} \Gamma\right)$.

Since $\Gamma / F \Gamma$ is finite, we can take $S$ to be finite. So $\left|\Gamma / F^{r} \Gamma\right| \leq\left|\left[S^{(r)}\right]_{F}\right| \leq|S|^{r}<\infty$, and $\Gamma / F^{r} \Gamma$ is finite, as desired.

Corollary 3.10. Suppose there is an $F^{r}$-spanning set for $\Gamma$ for some $r>0$ and $H \leq \Gamma$ is $F$-invariant. Then there is an $F^{s}$-spanning set for $H$ for some $s>0$. Furthermore if $A \subseteq H$ then $A$ is $F$-automatic in $\Gamma$ if and only $A$ is F-automatic in $H$.

Proof. By Theorem 3.9 there is a length function $\lambda$ for some $\left(\Gamma, F^{r}\right)$. One can check that $\lambda\lceil H$ is a length function for $\left(H,\left(F\lceil H)^{r}\right)\right.$. So again by Theorem 3.9 there is an $F^{s}$-spanning set for $H$ for some $s>0$.

For the "furthermore", note by the above and [2, Lemmas 5.6 and 5.7] there is $s$ such that there is an $F^{s}$-spanning set $\Sigma$ for $H$ and that is contained in an $F^{s}$-spanning set $\Sigma^{\prime}$ for $\Gamma$. The right-to-left direction then follows from [2, Proposition 6.8 (b)]. For the left-to-right, note that $\left\{\sigma \in \Sigma^{*}:[\sigma]_{F^{s}} \in A\right\}=\{\sigma \in$ $\left.\left(\Sigma^{\prime}\right)^{*}:[\sigma]_{F^{s}} \in A\right\} \cap \Sigma^{*}$ is the intersection of two regular languages, and is thus regular (see [7, Theorem 2.1]); so $A$ is $F$-automatic in $H$.

$\square$ Corollary 3.10

In fact when $\Gamma$ is a finitely generated abelian group, we can use the above to deduce a concrete and verifiable characterization of the existence of spanning sets.

Theorem 3.11. Suppose $\Gamma$ is a finitely generated abelian group. Then $\Gamma$ admits an $F^{r}$-spanning set for some $r>0$ if and only if the eigenvalues of $F \otimes_{\mathbb{Z}} \mathrm{id}_{\mathbb{C}}$ (viewed as a linear map on the $\mathbb{C}$-vector space $\Gamma \otimes_{\mathbb{Z}} \mathbb{C}$ ) all have modulus $>1$.

Proof. We first show that we can reduce to the torsion-free case. By the fundamental theorem of finitely generated abelian groups we may assume $\Gamma=\mathbb{Z}^{m} \times H$ where $H$ is a finite group. Since $F$ is injective we get that $F^{-1} H=H$; so $F$ induces an injective endomorphism $F^{\prime}: \mathbb{Z}^{m} \rightarrow \mathbb{Z}^{m}$ on the quotient $\Gamma / H \cong \mathbb{Z}^{m}$. Then $\Gamma$ has an $F$-spanning set if and only if $\left(\mathbb{Z}^{m}, F^{\prime}\right)$ does: it is not hard to verify that if $\Sigma$ is an $F$-spanning set for $\Gamma$ and $\pi: \Gamma \rightarrow \mathbb{Z}^{m}$ is the projection then $\pi(\Sigma)$ is an $F^{\prime}$-spanning set for $\mathbb{Z}^{m}$, and conversely if $\Sigma$ is an $F^{\prime}$-spanning set for $\mathbb{Z}^{m}$ then $\pi^{-1}(\Sigma)$ is an $F$-spanning set for $\Gamma$. Note further that under the identification $\Gamma \otimes_{\mathbb{Z}} \mathbb{C}=\mathbb{Z}^{m} \otimes_{\mathbb{Z}} \mathbb{C}$ we have that $F \otimes_{\mathbb{Z}} \mathrm{id}_{\mathbb{C}} \sim F^{\prime} \otimes_{\mathbb{Z}} \mathrm{id}_{\mathbb{C}}$, and in particular they have the same eigenvalues.

We may therefore assume $\Gamma=\mathbb{Z}^{m}$ for some $m$ and $F \in M_{m}(\mathbb{Z})$. 
( $\Longleftarrow$ ) By replacing $F$ with a power, we may assume $|\mu|>2$ for all $\mu \in \sigma(F)$. We show there exists a height function for $(\Gamma, F)$. Pick a basis $\left\{e_{1}, \ldots, e_{m}\right\}$ for $\mathbb{C}^{m}$ that puts $F$ in Jordan canonical form. Let $h: \mathbb{C}^{m} \rightarrow \mathbb{R}_{\geq 0}$ be the infinity norm associated to $\left\{e_{1}, \ldots, e_{m}\right\}$ : so $h\left(a_{1} e_{1}+\cdots+a_{m} e_{m}\right)=\max _{i}\left|a_{i}\right|$. We show that $h \vdash \mathbb{Z}^{m}$ is a height function for $(\Gamma, F)$. Triangle inequality and symmetry are clear; Northcott follows from the fact that all norms on a finite-dimensional space are equivalent (and in particular that our infinity norm is equivalent to the usual infinity norm on $\mathbb{C}^{m}$ ). It remains to check canonicity.

Fix some $\beta>1$ such that $\beta+1<|\mu|$ for all $\mu \in \sigma(F)$. Suppose $v=v_{1} e_{1}+\cdots+v_{m} e_{m} \in \mathbb{C}^{m} \backslash\{0\}$; write $F v=w_{1} e_{1}+\cdots+w_{m} e_{m}$. Fix $i$ such that $\left|v_{i}\right|=h(v)$. Depending on the structure of the Jordan blocks, we get that $w_{i}$ is either $\mu v_{i}+v_{i+1}$ or $\mu v_{i}$ (for $\mu \in \sigma(F)$ corresponding to $v_{i}$ ). In the first case, reverse triangle inequality yields

$$
h(F v) \geq\left|w_{i}\right| \geq|\mu|\left|v_{i}\right|-\left|v_{i+1}\right| \geq(|\mu|-1)\left|v_{i}\right|>\beta\left|v_{i}\right|=\beta h(v)
$$

and in the second case we get

$$
h(F v) \geq\left|w_{i}\right|=|\mu|\left|v_{i}\right|>\beta\left|v_{i}\right|=\beta h(v)
$$

So $h$ satisfies canonicity, as desired.

( $\Longrightarrow$ ) Suppose there is $\mu \in \sigma(F)$ with $|\mu| \leq 1$. There are two cases:

Case 1. Suppose there is $\mu \in \sigma(F)$ with $|\mu|<1$; the argument in this case is similar to Example 3.1. Since the same is true for all powers of $F$ it suffices to show that there is no $F$-spanning set. Pick a basis $\left\{e_{1}, \ldots, e_{m}\right\}$ for $\mathbb{C}^{m}$ that puts $F$ into Jordan canonical form; for $x \in \mathbb{C}^{m}$ we write

$$
x=\sum_{i=1}^{m} f_{i}(x) e_{i}
$$

so each $f_{i}: \mathbb{C}^{m} \rightarrow \mathbb{C}$ is linear. Pick some $e_{k}$ corresponding to the bottom-right of some Jordan block for $\mu$; so $f_{k}(F x)=\mu f_{k}(x)$ for $x \in \mathbb{C}^{m}$.

Suppose for contradiction we had an $F$-spanning set $\Sigma$. Let $M=\max \left\{\left|f_{k}(a)\right|: a \in \Sigma\right\}$. Then if

$$
y=\sum_{i=0}^{\ell} F^{i} a_{i}
$$

for $a_{i} \in \Sigma$ then

$$
\left|f_{k}(y)\right|=\left|\sum_{i=0}^{\ell} \mu^{i} f_{k}\left(a_{i}\right)\right| \leq \sum_{i=0}^{\ell}\left|\mu^{i} f_{k}\left(a_{i}\right)\right|=\sum_{i=0}^{\ell}|\mu|^{i}\left|f_{k}\left(a_{i}\right)\right| \leq M \sum_{i<\omega}|\mu|^{i}=M \frac{1}{1-|\mu|}
$$

So every $y \in \mathbb{Z}^{m}$ satisfies $\left|f_{k}(y)\right| \leq M \frac{1}{1-|\mu|}$. So since the integers are closed under doubling and $f_{k}$ is linear, we get that $f_{k}(y)=0$ for each $y \in \mathbb{Z}^{m}$. So $\mathbb{C}^{m}=\operatorname{span}_{\mathbb{C}} \mathbb{Z}^{m}$ is spanned by $\left\{e_{1}, \ldots, e_{m}\right\} \backslash\left\{e_{k}\right\}$, a contradiction.

Case 2. Suppose $|\mu| \geq 1$ for all $\mu \in \sigma(F)$; pick some $\mu \in \sigma(F)$ with $|\mu|=1$. We show that there is non-zero $a \in \mathbb{Z}^{m}$ such that $F^{i} a=F^{j} a$ for some $i \neq j$, and deduce that no power of $F$ admits a length function.

Let $p_{\mu}(x)$ be the minimal polynomial for $\mu$ over $\mathbb{Q}$. Note that if $\nu \in \mathbb{C}$ is a root of $p_{\mu}$ then so is $\nu^{-1}$. Indeed, this is true of $\mu$ since $\mu^{-1}=\bar{\mu}$ and $p_{\mu} \in \mathbb{Q}[x]$, and hence is true of all Aut $(\overline{\mathbb{Q}} / \mathbb{Q})$ conjugates of $\mu$. But $p_{\mu}$ divides the characteristic polynomial of $F$, and hence $p_{\mu}$ has no roots of modulus $<1$ by hypothesis. It follows that $p_{\mu}$ has no roots of modulus $>1$ either. That is, the roots of $p_{\mu}$ lie on the unit circle.

Now, $p_{\mu}(F)$ isn't invertible over $\mathbb{C}$, and hence isn't invertible over $\mathbb{Q}$; so $V:=\operatorname{ker}_{\mathbb{Q}}\left(p_{\mu}(F)\right)$ is a non-trivial $F$-invariant subspace of $\mathbb{Q}^{m}$. Furthermore $p_{\mu}(F\lceil V)=0$, so the minimal polynomial 
of $F \uparrow V$ is separable (since $p_{\mu}$ is); so $F \uparrow V$ is diagonalizable (over $\mathbb{C}$ ) with eigenvalues that are roots of $p_{\mu}$, and hence lie on the unit circle. In particular in the inner product induced by the eigenbasis for $F \uparrow V$ we get that $F \uparrow V$ is unitary.

Since $V$ is a non-trivial subspace of $\mathbb{Q}^{m}$ it contains a non-zero $a \in \mathbb{Z}^{m}$. So $F a \in V \cap \mathbb{Z}^{m}$ as well and $\|F a\|=\|a\|$ (where the norm is induced by the aforementioned inner product); inductively we get $\left\|F^{n} a\right\|=\|a\|$ and $F^{n} a \in \mathbb{Z}^{m}$ for all $n$. But $\|\cdot\|$ is equivalent to the Euclidean norm (since both are norms on a finite-dimensional space); so there are finitely many $b \in \mathbb{Z}^{m}$ with $\|b\|=\|a\|$. So $F^{i} a=F^{j} a$ for some $i \neq j$. By injectivity of $F$ we get $F^{i-j} a=a$, and hence that $\left(F^{n}\right)^{i-j} a=a$ for all $n$. It follows that the $F^{n}$-orbit of $a$ is finite for all $n>0$. Since $a$ has infinite order, Remark 3.6 yields that no power of $F$ admits a length function. Hence by Theorem $3.9 \Gamma$ does not admit an $F^{r}$-spanning set for any $r>0$.

Theorem 3.11

\section{A characterization of $F$-automaticity}

A useful characterization of classical automaticity is in terms of "finiteness of kernels". A version of this for $F$-automaticity is given in [2, Lemma 6.2]. Using length functions we are able to improve this result. First, here is what kernels mean in our setting, as introduced in Definition 6.1 of [2]. As before, we fix an abelian group $\Gamma$ with an injective endomorphism $F$.

Definition 4.1. Suppose $A \subseteq \Gamma$. Given $s_{0}, \ldots, s_{n-1} \in \Gamma$ we set

$$
A_{s_{0} \cdots s_{n-1}}(x)=\left\{x \in \Gamma: s_{0}+\cdots+F^{n-1} s_{n-1}+F^{n} x \in A\right\}
$$

(so $A_{\varepsilon}=A$ ). Given $S \subseteq \Gamma$, by the $(S, F)$-kernel of $A$ we mean the set $\left\{A_{\sigma}: \sigma \in S^{*}\right\}$.

Theorem 4.2. Suppose $\Gamma$ admits an $F^{r}$-spanning set for some $r>0$ (and so in particular that $\Gamma / F \Gamma$ is finite). Fix finite $S \subseteq \Gamma$ containing a representative of each coset of $F \Gamma$ in $\Gamma$. Then $A \subseteq \Gamma$ is $F$-automatic if and only if the $(S, F)$-kernel of $A$ is finite.

The big improvement here is that $S$ need not be a spanning set-being a complete set of representatives for $\Gamma / F \Gamma$ is a much weaker condition. A nice feature of this characterization is that, apart from the hypothesis on existence of a spanning set, $F$-automaticity of a set $A$ can be checked without references to higher powers of $F$.

Lemma 4.3. Let $\Gamma, F$, and $S$ be as in Theorem 4.2. Suppose $n>0$. The $(S, F)$-kernel of $A$ is finite if and only if the $\left(S^{(n)}, F^{n}\right)$-kernel of $A$ is finite.

Proof. The $\left(S^{(n)}, F^{n}\right)$-kernel is clearly contained in the $(S, F)$-kernel. Suppose the $(S, F)$-kernel is infinite.

Note that if $\sigma=s_{0} \cdots s_{k-1}, \tau=t_{0} \cdots t_{\ell-1} \in S^{*}$ and $a \in \Gamma$ then

$$
\begin{aligned}
a \in\left(A_{\sigma}\right)_{\tau} & \Longleftrightarrow t_{0}+\cdots+F^{\ell-1} t_{\ell-1}+F^{\ell} a \in A_{\sigma} \\
& \Longleftrightarrow s_{0}+\cdots+F^{k-1} s_{k-1}+F^{k}\left(t_{0}+\cdots+F^{\ell-1} t_{\ell-1}+F^{\ell} a\right) \in A \\
& \Longleftrightarrow s_{0}+\cdots+F^{k-1} s_{k-1}+F^{k} t_{0}+\cdots+F^{k+\ell-1} t_{\ell-1}+F^{k+\ell} a \in A \\
& \Longleftrightarrow a \in A_{\sigma \tau}
\end{aligned}
$$

So $\left(A_{\sigma}\right)_{\tau}=A_{\sigma \tau}$; in particular, if $A_{\sigma \tau} \neq A_{\sigma^{\prime} \tau}$ then $A_{\sigma} \neq A_{\sigma^{\prime}}$.

Since the set of all $A_{\sigma}$ is infinite, there is $0 \leq i<n$ such that the set of $A_{\sigma}$ with $|\sigma| \equiv i(\bmod n)$ is infinite. Now, as $S$ is finite, this means there is $\tau \in S^{*}$ of length $i$ such that $\left\{A_{\rho \tau}: \rho \in S^{*},|\rho| \in n \mathbb{Z}\right\}$ is infinite. It follows by the above observation that $\left\{A_{\rho}: \rho \in S^{*},|\rho| \in n \mathbb{Z}\right\}$ is infinite, and so the $\left(S^{(n)}, F^{n}\right)$-kernel is infinite.

$\square$ Lemma 4.3

Lemma 4.4. Suppose $\Gamma$ admits a length function $\lambda$ for $(\Gamma, F)$ with associated constants $C, D, E$ such that $C \geq D$. Suppose $S, T \subseteq \Gamma$ are finite sets both containing a representative of each coset of $F \Gamma$. Then $A$ has finite $(S, F)$-kernel if and only if it has finite $(T, F)$-kernel. 
Proof. Let $N=\max \{\lambda(s-t): s \in S, t \in T\}$. Suppose the $(T, F)$-kernel of $A$ is finite.

Take an element $A_{\sigma}$ of the $(S, F)$-kernel of $A$, say with $\sigma=s_{0} \cdots s_{n-1} \in S^{*}$. By the proof of Theorem 3.9 there is $\tau=t_{0} \ldots t_{n-1} \in T^{*}$ such that

$$
s_{0}+\cdots+F^{n-1} s_{n-1} \equiv t_{0}+\cdots+F^{n-1} t_{n-1} \quad\left(\bmod F^{n}(\Gamma)\right)
$$

So $A_{\tau}$ lies in the $(T, F)$-kernel of $A$. Let

$$
\delta=F^{-n}\left(\left(s_{0}-t_{0}\right)+\cdots+F^{n-1}\left(s_{n-1}-t_{n-1}\right)\right)
$$

so for $a \in \Gamma$ we have

$$
\begin{aligned}
a+\delta \in A_{\tau} & \Longleftrightarrow t_{0}+\cdots+F^{n-1} t_{n-1}+F^{n}(a+\delta) \in A \\
& \Longleftrightarrow t_{0}+\cdots+F^{n-1} t_{n-1}+F^{n} a+\left(s_{0}-t_{0}\right)+\cdots+F^{n-1}\left(s_{n-1}-t_{n-1}\right) \in A \\
& \Longleftrightarrow s_{0}+\cdots F^{n-1} s_{n-1}+F^{n} a \in A \\
& \Longleftrightarrow a \in A_{\sigma}
\end{aligned}
$$

So $A_{\sigma}=A_{\tau}-\delta$. Hence to show that the $(S, F)$-kernel of $A$ is finite it suffices to show that $\delta$ can take on one of only finitely many values (as $\sigma, \tau$ vary). We show that $\lambda(\delta)$ is bounded in terms of $S, T$, which suffices by the Northcott property.

We first bound $\lambda\left(F^{n} \delta\right)=\lambda\left(\left(s_{0}-t_{0}\right)+\cdots+F^{n-1}\left(s_{n-1}-t_{n-1}\right)\right)$. Since $\lambda\left(s_{0}-t_{0}\right) \leq N$ and $\lambda\left(F\left(s_{1}-t_{1}\right)\right) \leq$ $C N$, the ultrametric inequality yields

$$
\lambda\left(\left(s_{0}-t_{0}\right)+F\left(s_{1}-t_{1}\right)\right) \leq D C N
$$

Since $\lambda\left(F^{2}\left(s_{2}-t_{2}\right)\right) \leq C^{2} N$, another application of ultrametric inequality yields

$$
\lambda\left(\left(s_{0}-t_{0}\right)+F\left(s_{1}-t_{1}\right)+F^{2}\left(s_{2}-t_{2}\right)\right) \leq D C^{2} N
$$

(since by hypothesis $C \geq D$ ). Continuing inductively, we find that $\lambda\left(F^{n} \delta\right) \leq D C^{n-1} N$. Hence by canonicity we get that $\lambda(\delta) \leq E C^{-1} D N$.

$\square$ Lemma 4.4

Proof of Theorem 4.2. Suppose $\Gamma$ admits an $F^{n}$-spanning set $\Sigma$ for some $n>0$. By Proposition $3.8 \lambda_{\Sigma}$ is a length function for $\left(\Gamma, F^{n}\right)$ with associated constants $C, D, E$ such that $C \geq D$. Suppose $A \subseteq \Gamma$. Then by the previous two lemmas, the $(S, F)$-kernel of $A$ is finite if and only if the $\left(S^{(n)}, F^{n}\right)$-kernel of $A$ is, which occurs if and only if the $\left(\Sigma, F^{n}\right)$-kernel of $A$ is. (Note that an $F^{n}$-spanning set must contain a representative of every coset of $F \Gamma$.) But by [2, Lemma 6.2] this is equivalent to $A$ being $F$-automatic, as desired.

As an illustration of the usefulness of this characterization we give quick proof of [2, Lemma 6.7(a)]: that if a spanning set exists then the equivalence relation of "representing the same element" on strings is characterized by a finite automaton.

Corollary 4.5. Suppose $\Sigma$ is an F-spanning set. Then $\left\{(\sigma, \tau) \in\left(\Sigma^{2}\right)^{*}:[\sigma]_{F}=[\tau]_{F}\right\}$ is a regular language over $\left(\Sigma^{2}\right)^{*}$.

Proof. By Proposition 2.6 this is equivalent to $\Delta=\{(a, a): a \in \Gamma\} \subseteq \Gamma^{2}$ being $F$-automatic. Fix a set $S$ containing exactly one representative of each coset of $F \Gamma$ in $\Gamma$; we will show that the $\left(S^{2}, F\right)$-kernel of $\Delta$ is finite. Note that if $s_{0} \cdots s_{n-1}, t_{0} \cdots t_{n-1} \in S^{(n)}$ are unequal, say with $i$ minimal such that $s_{i} \neq t_{i}$, then since $s_{i} \not \equiv t_{i}(\bmod F \Gamma)$ and $F$ is injective we get that $s_{0}+\cdots+F^{n-1} s_{n-1} \not \equiv t_{0}+\cdots+F^{n-1} t_{n-1}$ $\left(\bmod F^{i+1} \Gamma\right)$. So $s_{0}+\cdots+F^{n-1} s_{n-1} \not \equiv t_{0}+\cdots+F^{n-1} t_{n-1}\left(\bmod F^{n} \Gamma\right)$, and thus $s_{0}+\cdots+F^{n-1} s_{n-1}+F^{n} a \neq$ $t_{0}+\cdots+F^{n-1} t_{n-1}+F^{n} b$ for any $a, b \in \Gamma$. So $\Delta_{(\sigma, \tau)}$ is empty if $(\sigma, \tau) \in\left(S^{2}\right)^{*}$ and $\sigma \neq \tau$. Furthermore if $\sigma=\tau$ then by injectivity of $F$ we get $\Delta_{(\sigma, \tau)}=\Delta$. So the $\left(S^{2}, F\right)$-kernel of $\Delta$ contains two elements.

$\square$ Corollary 4.5 


\section{A characterization of $F$-sparsity}

Recall that a language $L \subseteq \Sigma^{*}$ is sparse if it is regular and $|\{\sigma \in L:|\sigma| \leq x\}|$ grows polynomially in $x$. See the beginning of [2, Section 7] for a brief overview of sparsity.

Fix $(\Gamma, F)$ an abelian group equipped with an injective endomorphism. In [2] Bell and Moosa adapt the notion of sparsity to the setting of $F$-automatic sets.

Definition 5.1. A subset $A \subseteq \Gamma$ is $F$-sparse if there is an $F^{r}$-spanning set $\Sigma$ for some $r>0$ and a sparse $L \subseteq \Sigma^{*}$ such that $A=[L]_{F^{r}}$.

While the definition of $F$-sparsity is sufficient for the purposes of [2], it is cumbersome in some contexts. In particular, in order to show a set is not sparse one needs to check every possible set of representatives in every possible spanning set for every possible power of $F$. For example, it is not immediate from the definition that $\Gamma$ itself isn't $F$-sparse.

In this section we will give a characterization of $F$-sparsity in terms of length functions, and deduce from that some natural properties of $F$-sparsity. We will use the following characterization of sparsity:

Fact 5.2 ([2, Proposition 7.1]). $L \subseteq \Sigma^{*}$ is sparse if and only if it is a finite union of sets of the form $v_{0} w_{1}^{*} v_{1} \cdots v_{n-1} w_{n}^{*} v_{n}$ where $v_{0}, \ldots, v_{n}, w_{1}, \ldots, w_{n} \in \Sigma^{*}$.

We call languages of the form $v_{0} w_{1}^{*} v_{1} \cdots v_{n-1} w_{n}^{*} v_{n}$ simple sparse. By Fact 5.2 an $F$-sparse set is a finite union of sets of the form $[L]_{F^{r}}$ where $L$ is a simple sparse language. The following further simplification in the structure of $F$-sparse sets will be useful both for proving basic closure properties below, and for studying stability in the next section.

Proposition 5.3. Suppose $A \subseteq \Gamma$ is $F$-sparse. There is $s_{0} \in \mathbb{N}$ such that for all $s \in s_{0} \mathbb{N}$ we can write $A$ as a finite union of translates of sets of the form $\left[a_{1}^{*} \cdots a_{n}^{*}\right]_{F^{s}}$ where $a_{1}, \ldots, a_{n} \in \Gamma$.

Proof. By Fact 5.2 we can write $A$ as a finite union of sets of the form $\left[v_{0} w_{1}^{*} v_{1} \cdots w_{n}^{*} v_{n}\right]_{F^{r}}$ for some strings $v_{i}, w_{i} \in \Gamma^{*}$. By replacing $F$ with $F^{r}$ we may assume $r=1$. Let $s_{0}$ be the least common multiple of all the $\left|w_{i}\right|$.

In the case where $\Gamma=\mathbb{Z}^{m}$ and $F$ is multiplication by some $d>0$, an intermediate result in the proof of [5, Lemma 3.5] is that we can write $A$ as a finite union of translates of sets of the form $\left[\tau_{1}^{*} \cdots \tau_{n}^{*}\right]_{F}$ where $\tau_{1}, \ldots, \tau_{n} \in \Gamma^{*}$ all have length $s_{0}$. In fact with little additional effort the proof of this generalizes to our setting, and can be made to show that if $s \in s_{0} \mathbb{N}$ then we may take all $\tau_{i}$ to have length $s$ rather than $s_{0}$. But if we let $a_{i}=\left[\tau_{i}\right]_{F}$ then $\left[\tau_{1}^{*} \cdots \tau_{n}^{*}\right]_{F}=\left[a_{1}^{*} \cdots a_{n}^{*}\right]_{F s}$; so we have written $A$ in the desired form.

We note some closure properties:

\section{Proposition 5.4.}

1. For any $A \subseteq \Gamma$ and $r>0, A$ is $F$-sparse if and only if it is $F^{r}$-sparse.

2. If $A, B \subseteq \Gamma$ are $F$-sparse then so is $A \cup B$.

3. If $A \subseteq \Gamma$ is $F$-sparse and $X \subseteq \Gamma$ is $F$-automatic then $A \cap X$ is $F$-sparse.

4. If $A, B \subseteq \Gamma$ are $F$-sparse then so is $A+B$.

Proof.

1. The right-to-left is by definition; for the left-to-right, take an $F^{r}$-spanning set $\Sigma$ for some $r>0$ and a sparse language $L \subseteq \Sigma^{*}$ such that $A=[L]_{F^{r}}$. Recall by [2, Lemma 5.7] that $\Sigma^{\prime}=\left[\Sigma^{(s)}\right]_{F^{r}}$ is an $F^{r s}$-spanning set. Given $\sigma \in \Sigma^{*}$ with $s|| \sigma \mid$ we can associate $\sigma^{\prime} \in\left(\Sigma^{\prime}\right)^{*}$ as follows: write $\sigma=\sigma_{1} \cdots \sigma_{\frac{|\sigma|}{s}}$ with each $\sigma_{i} \in \Sigma^{(s)}$, and set $\sigma^{\prime}=\left[\sigma_{1}\right]_{F^{r}} \cdots\left[\sigma_{\frac{|\sigma|}{s}}\right]_{F^{r}}$. So $[\sigma]_{F^{r}}=\left[\sigma^{\prime}\right]_{F^{r s}}$, and $\left|\sigma^{\prime}\right|=\frac{|\sigma|}{s}$.

Note now that

$$
\left.A=[L]_{F^{r}}=\left[L 0^{*} \cap\left(\Sigma^{(s)}\right)\right)^{*}\right]_{F^{r}}=[\underbrace{\left\{\sigma^{\prime}: \sigma \in L 0^{*} \cap \Sigma^{(s \mathbb{N})}\right\}}_{L^{\prime}}]_{F^{r s}}
$$


Since regular languages are closed under intersection (see [7, Theorem 2.1]), we get that $L 0^{*} \cap\left(\Sigma^{s}\right)^{*}$ is regular; one can then use a DFA that recognizes $L 0^{*} \cap\left(\Sigma^{s}\right)^{*}$ to construct a DFA that recognizes $L^{\prime}$. By Fact 5.2 $L 0^{*}$, and hence $L 0^{*} \cap\left(\Sigma^{s}\right)^{*}$, is a sparse language; thus since $\left|\sigma^{\prime}\right|=\frac{|\sigma|}{s}$ we get that $L^{\prime}$ is sparse. So $A=\left[L^{\prime}\right]_{F^{r s}}$ is $F^{s}$-sparse.

2. Take an $F^{r}$-spanning set $\Sigma$ and an $F^{s}$-spanning set $\Theta$ with sparse languages $L_{1} \subseteq \Sigma^{*}$ and $L_{2} \subseteq \Theta^{*}$ such that $A=\left[L_{1}\right]_{F^{r}}$ and $B=\left[L_{2}\right]_{F^{s}}$. By the argument given in the proof of (1), we may assume $r=s$. By [2, Lemma 5.6], there is an $F^{r}$-spanning set $\Omega$ containing $\Sigma \cup \Theta$. Then $L_{1} \cup L_{2}$ is a sparse language in $\Omega^{*}$, and $A \cup B=\left[L_{1} \cup L_{2}\right]_{F^{r}}$. So $A \cup B$ is $F$-sparse.

3. Take an $F^{r}$-spanning set $\Sigma$ for some $r>0$ and a sparse language $L \subseteq \Sigma^{*}$ such that $A=[L]_{F^{r}}$. By Proposition 2.6 $\left\{\sigma \in \Sigma^{*}:[\sigma]_{F^{r}} \in X\right\}$ is regular. So if $L^{\prime}=\left\{\sigma \in L:[\sigma]_{F^{r}} \in X\right\}$ then $L^{\prime}$ is regular (as the intersection of two regular languages; see [7, Theorem 2.1]) and thus sparse (as it's contained in $L$ ). But $\left[L^{\prime}\right]_{F^{r}}=A \cap X$; so $A \cap X$ is $F$-sparse.

4. We first check the case where $B=\{\gamma\}$ is a singleton. Take an $F^{r}$-spanning set $\Sigma$ for some $r>0$ and a sparse language $L \subseteq \Sigma^{*}$ such that $A=[L]_{F^{r}}$. By [2, Lemma 5.6] there is an $F^{r}$-spanning set $\Sigma^{\prime} \supseteq \Sigma$ that contains $a+\gamma$ for every $a \in \Sigma$. Given $\sigma=a_{1} \cdots a_{|\sigma|} \in \Sigma^{*}$ non-empty let $\sigma_{\gamma}=$ $\left(a_{1}+\gamma\right) a_{2} \cdots a_{|\sigma|} \in\left(\Sigma^{\prime}\right)^{*}$; so $\left[\sigma_{\gamma}\right]_{F^{r}}=[\sigma]_{F^{r}}+\gamma$. It is routine to check that sparsity of $L$ implies sparsity of $L_{\gamma}:=\left\{\left[\sigma_{\gamma}\right]_{F^{r}}: \sigma \in L\right\} \subseteq\left(\Sigma^{\prime}\right)^{*}$. So $A+\gamma=\left[L_{\gamma}\right]_{F^{r}}$ is $F$-sparse.

We now do the general case. By Proposition 5.3 there is $s$ such that there is an $F^{s}$-spanning set and such that both $A$ and $B$ can be written as a finite union of translates of sets of the form $\left[a_{1}^{*} \cdots a_{n}^{*}\right]_{F^{s}}$. Distributing, it suffices to check the case $A=\gamma+\left[a_{1}^{*} \cdots a_{n}^{*}\right]_{F}$ and $B=\gamma^{\prime}+\left[b_{1}^{*} \cdots b_{n^{\prime}}^{*}\right]_{F^{s}}$. By the first case, we may assume $\gamma=\gamma^{\prime}=0$. Given $\sigma \in a_{1}^{*} \cdots a_{n}^{*}$ and $\tau \in b_{1}^{*} \cdots b_{n^{\prime}}^{*}$ we let $\sigma \oplus \tau \in \Gamma^{*}$ denote the string obtained by characterwise addition; so $[\sigma \oplus \tau]_{F^{s}}=[\sigma]_{F^{s}}+[\tau]_{F^{s}}$. Then

$$
a_{1}^{*} \cdots a_{n}^{*} \oplus b_{1}^{*} \cdots b_{n^{\prime}}^{*}=\left(a_{1}+b_{1}\right)^{*}\left(a_{2}^{*} \cdots a_{n}^{*}+b_{1}^{*} \cdots b_{n^{\prime}}^{*}\right) \cup\left(a_{1}+b_{1}\right)^{*}\left(a_{1}^{*} \cdots a_{n}^{*}+b_{2}^{*} \cdots b_{n^{\prime}}^{*}\right)
$$

and is thus sparse by an inductive argument on $\left(n, n^{\prime}\right)$. So $A+B=\left[a_{1}^{*} \cdots a_{n}^{*} \oplus b_{1}^{*} \cdots b_{n^{\prime}}^{*}\right]_{F^{s}}$ is $F$ sparse.

$\square$ Proposition 5.4

We now work towards a characterization of $F$-sparsity using length functions.

Definition 5.5. Suppose $A \subseteq \Gamma$ and $\lambda$ is a length function for $(\Gamma, F)$. We let $f_{A, \lambda}(x)=|\{a \in A: \lambda(a) \leq x\}|$, and we say $A$ is $\lambda$-sparse if $f_{A, \lambda}(x) \in O\left(\log (x)^{d}\right)$ for some $d \in \mathbb{N}$.

To explain the jump from "polynomial in $x$ " to "polynomial in $\log (x)$ ", recall that the length function associated to a spanning set $\Sigma$ was defined by raising 2 to the length of the shortest word representing the input; the logarithm is there to undo the exponentiation. Indeed, if $\lambda=\lambda_{\Sigma}$ is the length function of Definition 3.7 associated to an $F^{r}$-spanning set $\Sigma$ for some $r>0$ then $A$ is $\lambda$-sparse if and only if $\left|\left\{a \in A: a \in\left[\Sigma^{(x)}\right]_{F^{r}}\right\}\right|$ grows polynomially in $x$.

For our characterization of $F$-sparsity we will need the following reverse ultrametric inequality:

Lemma 5.6. If $\lambda$ is a length function for $(\Gamma, F)$ with associated constants $C, D, E$ and $a, b \in \Gamma$ satisfy $\lambda(b)<D^{-1} \lambda(a)$ then $\lambda(a+b) \geq D^{-1} \lambda(a)$.

Proof. Otherwise $\lambda(a)>D \max (\lambda(b), \lambda(a+b))=D \max (\lambda(-b), \lambda(a+b)) \geq \lambda(a)$.

Lemma 5.6

We will also require the following observation relating the length of a string to the length of the group element it represents:

Lemma 5.7. Suppose $\lambda$ is a length function for $(\Gamma, F)$ with associated constants $C, D, E$; suppose $\Sigma$ is a finite subset of $\Gamma$. Then there is $M>0$ such that $\lambda\left([\sigma]_{F}\right) \leq M C^{|\sigma|}$ for all $\sigma \in \Sigma^{*}$.

Proof. Pick $r \in \mathbb{N}$ such that $C^{r} \geq D$, and let $K=\max \left\{\lambda\left([\sigma]_{F}\right): \sigma \in \Sigma^{(r)}\right\}$.

We show by induction on $k \geq 1$ that if $\sigma \in \Sigma^{(k r)}$ then $\lambda\left([\sigma]_{F}\right) \leq D C^{(k-1) r} K$. The base case is just the definition of $K$. For the induction step, write $\sigma=\sigma_{1} \sigma_{2}$ where $\left|\sigma_{2}\right|=r$. Then by the induction hypothesis $\lambda\left(\left[\sigma_{1}\right]_{F}\right) \leq D C^{(k-2) r} K$, and by canonicity we get that $\lambda\left(F^{(k-1) r}\left[\sigma_{2}\right]_{F}\right) \leq C^{(k-1) r} \lambda\left(\left[\sigma_{2}\right]_{F}\right) \leq C^{(k-1) r} K$. So 
by the ultrametric inequality we get that $\lambda\left([\sigma]_{F}\right)=\lambda\left(\left[\sigma_{1}\right]_{F}+F^{(k-1) r}\left[\sigma_{2}\right]_{F}\right) \leq D \max \left(C^{(k-1) r} K, D C^{(k-2) r} K\right)=$ $D C^{(k-1) r}$ (since $C^{r} \geq D$ ).

Now, for any $\sigma \in \Sigma^{*}$ we can pad by some string of zeroes $\tau$ of length $<r$ to get that $|\sigma \tau| \in r \mathbb{Z}$, at which point we get $\lambda\left([\sigma]_{F}\right)=\lambda\left([\sigma \tau]_{F}\right) \leq D C^{|\sigma \tau|-r} K \leq D C^{|\sigma|} K$.

$\square$ Lemma 5.7

Lemma 5.8. Suppose $\Sigma$ is an $F^{r}$-spanning set for some $r>0$. Suppose $L \subseteq \Sigma^{*}$ is regular and $\preceq$ is a linear ordering on $\Sigma$ (which induces a length-lexicographical ${ }^{2}$ ordering on $\Sigma^{*}$ which we also denote by $\preceq$ ). Let

$$
\widetilde{L}=\left\{\sigma \in L: \sigma \preceq \tau \text { for all } \tau \in L \text { such that }[\sigma]_{F^{r}}=[\tau]_{F^{r}}\right\}
$$

Then $\widetilde{L}$ is regular.

Proof. Be replacing $F$ with $F^{r}$ we may assume $r=1$. Let $K \subseteq\left(\Sigma^{2}\right)^{*}$ be the set of $\left(\begin{array}{l}\sigma \\ \tau\end{array}\right)$ such that

- $\sigma, \tau \in L$;

- $[\sigma]_{F}=[\tau]_{F} ;$ and

- $\tau \prec \sigma$ or $\tau$ ends in a 0 .

So if $\sigma \in L$ then $\left(\begin{array}{c}\sigma \\ \tau\end{array}\right) \in K$ if and only if $\tau$ (with possibly the trailing 0 removed) witnesses that $\sigma \notin \widetilde{L}$; so $\widetilde{L}=L \backslash \pi(K)$, where $\pi:\left(\Sigma^{2}\right)^{*} \rightarrow \Sigma^{*}$ is projection to the first coordinate. So since $L$ is regular it suffices to show that $\pi(K)$ is regular (since by [7, Theorems 2.1 and 2.2] regular languages are closed under Boolean combinations).

Note that $K$ is regular: this is because $L \times L$, equality, ending in 0 , and $\prec$ are regular, and so $K$ is a Boolean combination of regular languages. Fix a DFA $\left(\Sigma^{2}, Q, q_{0}, \Omega, \delta\right)$ for $K$; we use this to construct an NFA $\left(\Sigma, Q^{\prime}, q_{0}^{\prime}, \Omega^{\prime}, \delta^{\prime}\right)$ recognizing $\pi(K)$. We let $Q^{\prime}=Q, q_{0}^{\prime}=q_{0}$, and $\Omega^{\prime}=\Omega$. For the transition function we set

$$
\delta^{\prime}(q, a)=\left\{\delta\left(q,\left(\begin{array}{l}
a \\
b
\end{array}\right)\right): b \in \Sigma\right\}
$$

Then $\sigma$ is accepted by our NFA if and only if there is $\tau \in \Sigma^{*}$ with $|\tau|=|\sigma|$ such that $\delta\left(q_{0},\left(\begin{array}{c}\sigma \\ \tau\end{array}\right)\right) \in \Omega$; i.e. such that $\left(\begin{array}{c}\sigma \\ \tau\end{array}\right) \in K$. So our NFA recognizes $\pi(K)$, and $\pi(K)$ is regular. So $\widetilde{L}$ is regular. $\quad \square$ Lemma 5.8

Theorem 5.9. Suppose $\lambda$ is a length function for some $\left(\Gamma, F^{r}\right)$. Then $A \subseteq \Gamma$ is $F$-sparse if and only if it is $F$-automatic and $\lambda$-sparse.

Proof. By Proposition 5.4 (1) it suffices to check the case $r=1$; so assume $\lambda$ is a length function for $(\Gamma, F)$. Let $C, D, E$ be the constants associated to $\lambda$.

( $\Longrightarrow$ ) Suppose $A$ is $F$-sparse; so there is some $F^{s}$-spanning set $\Sigma$ and some sparse $L \subseteq \Sigma^{*}$ such that $A=[L]_{F^{s}}$. We get by [2, Proposition 6.8(b)] that $A$ is $F$-automatic; it remains to show that $A$ is $\lambda$-sparse. Since $\lambda$ is also a length function for $\left(\Gamma, F^{s}\right)$ (see Remark 3.4), we can replace $F$ with $F^{s}$ and thus assume that $s=1$. By Fact $5.2 L$ is a finite union of simple sparse languages. One can verify that a finite union of $\lambda$-sparse sets is $\lambda$-sparse; it thus suffices to check the case where $L$ is simple sparse, say $L=v_{0} w_{1}^{*} \cdots v_{n-1} w_{n}^{*} v_{n}$ with $u_{i}, v_{i} \in \Sigma^{*}$. We apply induction on $n$; the base case $n=0$ is trivial.

For the induction step, we have two cases.

Case 1. Suppose $\left[w_{n}^{*} v_{n}\right]_{F}$ is finite. Then

$$
A=\bigcup_{a \in\left[w_{n}^{*} v_{n}\right]_{F}}\left[v_{0} w_{1}^{*} \cdots w_{n-1}^{*} v_{n-1} a\right]_{F}
$$

and by the induction hypothesis each $\left[v_{0} w_{1}^{*} \cdots w_{n-1}^{*} v_{n-1} a\right]_{F}$ is $\lambda$-sparse. So $A$ is $\lambda$-sparse.

\footnotetext{
${ }^{2}$ Recall that $\sigma \preceq \tau$ in the length-lexicographical order on $\Sigma^{*}$ induced by $\preceq$ if $|\sigma|<|\tau|$ or if $|\sigma|=|\tau|$ and $\sigma$ precedes $\tau$ in the lexicographical ordering induced by $\preceq$.
} 
Case 2. Suppose $\left[w_{n}^{*} v_{n}\right]_{F}$ is infinite.

Claim 5.10. There is $M>0$ and $i \in \mathbb{N}$ such that if $k_{n}>i$ then $\lambda\left(\left[v_{0} w_{1}^{k_{1}} \cdots w_{n}^{k_{n}} v_{n}\right]_{F}\right) \geq$

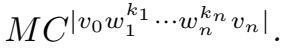

Proof. Our strategy will be to write

$$
\underbrace{\left[v_{0} w_{1}^{k_{1}} \cdots w_{n}^{k_{n}} v_{n}\right]_{F}}_{a}=\left[v_{0} w_{1}^{k_{1}} \cdots w_{n-1}^{k_{n-1}} v_{n-1} w_{n}^{k_{n}-i}\right]_{F}+\underbrace{F^{\left|v_{0} w_{1}^{k_{1}} \cdots w_{n-1}^{k_{n}-1} v_{n-1} w_{n}^{k_{n-i}}\right|}\left[w_{n}^{i} v_{n}\right]_{F}}_{b}
$$

and then use the reverse ultrametric inequality to show that $\lambda(a)$ is not much less than $\lambda(b)$.

Let $M_{0}$ be as in Lemma 5.7. Then by Northcott property there is some $i$ such that $\lambda\left(\left[w_{n}^{i} v_{n}\right]_{F}\right)>$ $E D M_{0}$. Suppose $k_{1}, \ldots, k_{n} \in \mathbb{N}$ with $k_{n} \geq i$; to avoid notational clutter we abbreviate $\sigma=$ $v_{0} w_{1}^{k_{1}} \cdots w_{n-1}^{k_{n-1}} v_{n-1} w_{n}^{k_{n}-i}$. We then wish to show that $\lambda\left(\left[\sigma w_{n}^{i} v_{n}\right]_{F}\right)$ is not much less than $\lambda\left(F^{|\sigma|}\left[w_{n}^{i} v_{n}\right]_{F}\right)$.

Note that

$$
\lambda\left(F^{|\sigma|}\left[w_{n}^{i} v_{n}\right]_{F}\right) \geq E^{-1} C^{|\sigma|} \lambda\left(\left[w_{n}^{i} v_{n}\right]_{F}\right)>C^{|\sigma|} D M_{0} \geq D \lambda\left([\sigma]_{F}\right)
$$

by hypothesis on $M_{0}$. So by the reverse ultrametric inequality we get that

$$
\lambda\left(\left[\sigma w_{n}^{i} v_{n}\right]_{F}\right)=\lambda\left([\sigma]_{F}+F^{|\sigma|}\left[w_{n}^{i} v_{n}\right]_{F}\right) \geq D^{-1} \lambda\left(F^{|\sigma|}\left[w_{n}^{i} v_{n}\right]_{F}\right) \geq D^{-1} E^{-1} C^{|\sigma|} \lambda\left(\left[w_{n}^{i} v_{n}\right]_{F}\right)
$$

Let $M=D^{-1} E^{-1} C^{-\left|w_{n}^{i} v_{n}\right|} \lambda\left(\left[w_{n}^{i} v_{n}\right]_{F}\right)$. Then if $k_{n} \geq i$ then $\lambda\left(\left[v_{0} w_{1}^{k_{1}} \cdots w_{n}^{k_{n}} v_{n}\right]_{F}\right) \geq M C^{\left|v_{0} w_{1}^{k_{1}} \cdots w_{n}^{k_{n}} v_{n}\right|}$, as desired.

$\square$ Claim 5.10

Now, we can write

$$
L=\left\{v_{0} w_{1}^{*} \cdots w_{n}^{k_{n}} v_{n}: k_{n} \geq i\right\} \cup \bigcup_{j<i} v_{0} w_{1}^{*} \cdots w_{n-1}^{*} v_{n-1} w_{n}^{j} v_{n}
$$

By the induction hypothesis each $\left[v_{0} w_{1}^{*} \cdots w_{n-1}^{*} v_{n-1} w_{n}^{j} v_{n}\right]_{F}$ is $\lambda$-sparse; it remains to check the case $k_{n} \geq i$.

If $\tau=v_{0} w_{1}^{k_{1}} \cdots w_{n}^{k_{n}} v_{n}$ with $k_{n} \geq i$ and $\tau$ satisfies $\lambda\left([\tau]_{F}\right) \leq x$ then by the claim $M C^{|\tau|} \leq x$, and thus $|\tau| \leq \log (C)^{-1}(\log (x)-\log (M))$. But by hypothesis there are $d, K$ such that eventually $|\{\tau \in L:|\tau| \leq y\}| \leq K y^{d}$. So if $x$ is sufficiently large there are at most $K\left(\log (C)^{-1}(\log (x)-\right.$ $\log (M))^{d} \in O\left(\log (x)^{d}\right)$ strings $\tau \in L$ satisfying $\lambda\left([\tau]_{F}\right) \leq x$; so $A$ is $\lambda$-sparse.

( $\Longleftarrow$ ) Let $\Sigma$ be an $F^{s}$-spanning set for some $s>0$. Note first that $A$ is $\lambda_{\Sigma}$-sparse. Indeed, by Lemma 5.7 (applied to $\left.\left(\Gamma, F^{s}\right)\right)$ there is $M>0$ such that $\lambda_{\Sigma}\left([\sigma]_{F^{s}}\right) \leq M C^{|\sigma|}$ for all $\sigma \in \Sigma^{*}$. So if $\lambda_{\Sigma}(a) \leq x$ then $\lambda(a) \leq M C^{\log _{2}(x)}=M x^{\log _{2}(C)}$; thus $f_{A, \lambda_{\Sigma}}(x) \leq f_{A, \lambda}\left(M x^{\log _{2}(C)}\right) \in O\left((\log (x))^{d}\right)$ for some $d$, and $A$ is $\lambda_{\Sigma}$-sparse.

Let $L=\left\{\sigma \in \Sigma^{*}:[\sigma]_{F^{s}} \in A\right\}$; so $L$ is regular by Proposition 2.6. Fix any total order $\preceq$ on $\Sigma$, and let $\widetilde{L}$ be as in Lemma 5.8; so $\widetilde{L}$ is also regular. Note also that $[\cdot]_{F s}$ is a bijection $\widetilde{L} \rightarrow A$ such that $\lambda_{\Sigma}\left([\sigma]_{F^{s}}\right)=2^{|\sigma|}$ : indeed, if there were a shorter $\tau \in \Sigma^{*}$ with $[\sigma]_{F^{s}}=[\tau]_{F^{s}}$ then $\tau \in L$, and thus $\tau$ witnesses that $\sigma \notin \widetilde{L}$. It follows that $\widetilde{L}$ is sparse: since $A$ is $\lambda_{\Sigma}$-sparse, we get that

$$
|\{\sigma \in \widetilde{L}:|\sigma| \leq x\}|=\left|\left\{a \in A: \lambda_{\Sigma}(a) \leq 2^{x}\right\}\right|=f_{A, \lambda_{\Sigma}}\left(2^{x}\right) \in O\left(\log \left(2^{x}\right)^{d}\right)=O\left(x^{d}\right)
$$

for some $d$. So $A=[\widetilde{L}]_{F^{s}}$ is $F$-sparse.

$\square$ Theorem 5.9

It follows that sparsity can be checked in any spanning set by looking at a set of "minimal" representations of elements of $A$; this can be seen as an analogue of Lemma 5.8 for sparsity.

Corollary 5.11. Suppose $\Sigma$ is an $F^{r}$-spanning set for some $r>0$. Suppose $L \subseteq \Sigma^{*}$ is regular and $\preceq$ is a linear ordering on $\Sigma$ (which we again identify with the induced length-lexicographic ordering on $\Sigma^{*}$ ). Let

$$
\widetilde{L}=\left\{\sigma \in L: \sigma \preceq \tau \text { for all } \tau \in L \text { such that }[\sigma]_{F^{r}}=[\tau]_{F^{r}}\right\}
$$

Then $[L]_{F^{r}}$ is $F$-sparse if and only if $\widetilde{L}$ is sparse. 
Proof. The right-to-left direction is by definition of $F$-sparsity. For the left-to-right, note by Lemma 5.8 that $\widetilde{L}$ is regular. Furthermore as in the proof of Theorem $5.9[\cdot]_{F^{r}}$ is a bijection $\widetilde{L} \rightarrow[L]_{F^{r}}$ with the property

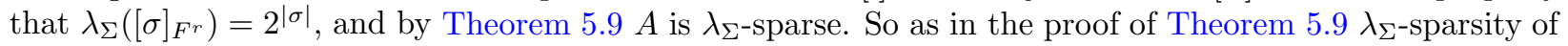
$A$ implies that $|\{\sigma \in \widetilde{L}:|\sigma| \leq x\}|$ grows polynomially in $x$. So $\widetilde{L}$ is sparse.

$\square$ Corollary 5.11

Another consequence is that if $A$ is contained in an $F$-invariant subgroup $H$ then sparsity of $A$ can be checked in $H$ :

Corollary 5.12. Suppose there is an $F^{r}$-spanning set for some $r>0$. Suppose $H \leq \Gamma$ is $F$-invariant and $A \subseteq H$. Then $A$ is $F$-sparse in $\Gamma$ if and only if $A$ is $F$-sparse in $H$.

Proof. For the right-to-left direction, note by Proposition 5.4 (1) that $A$ is $F^{r}$-sparse. So there is $s>0$ with an $F^{r s}$-spanning set $\Sigma$ for $H$ and $L \subseteq \Sigma^{*}$ sparse such that $A=[L]_{F^{r s}}$. Then using [2, Lemmas 5.6 and 5.7], since there is an $F^{r}$-spanning set for $\Gamma$ we may assume there is an $F^{r s}$-spanning set $\Sigma^{\prime}$ for $\Gamma$ containing $\Sigma$. Then $L \subseteq\left(\Sigma^{\prime}\right)^{*}$ witnesses that $A$ is $F$-sparse in $\Gamma$.

For the left-to-right direction, fix any length function $\lambda$ for some $\left(\Gamma, F^{s}\right)$ (using Theorem 3.9). One can check that $\lambda$ is a length function for $H$; so by Theorem 5.9 applied to $H$ it suffices to show that $A$ is $F$ automatic in $H$ and $\lambda$-sparse. By Theorem 5.9 applied to $\Gamma$ we get that $A$ is $F$-automatic in $\Gamma$ and $\lambda$-sparse. Then Corollary 3.10 yields that $A$ is $F$-automatic in $H$, and since $A \subseteq H$ we get that $\lambda$-sparsity in $\Gamma$ agrees with $\lambda$-sparsity in $H$. So $A$ is $F$-sparse in $H$.

$\square$ Corollary 5.12

We can now see that $\Gamma$ (and more generally any set containing an infinite $F$-invariant subgroup of $\Gamma$ ) is not $F$-sparse.

Corollary 5.13. Suppose $A \subseteq \Gamma$ is F-sparse.

1. A does not contain any coset of any infinite F-invariant subgroup.

2. If $\Gamma$ is finitely generated then $A$ does not contain any coset of any infinite subgroup.

Proof.

1. We first check the case $H=\Gamma$. Since $A$ is $F$-sparse there is an $F^{r}$-spanning set for some $r>0$; by Proposition 5.4 (1) we may assume that there is an $F$-spanning set $\Sigma$. By Lemma 3.5 we may assume the exceptional set of $\lambda_{\Sigma}$ contains only elements of finite $F$-orbit; say the new associated constants are $C, D, E$. Note that we can take $C=2$ : the constants obtained from Proposition 3.8 are $C=D=E=2$, and Lemma 3.5 doesn't require changing $C$. Note that there is $a \in \Sigma$ of infinite $F$-orbit: otherwise by Remark 3.6 all $a \in \Sigma$ would be of finite order as well, and thus all

$$
\mathbb{Z}[F] a=\left\{\sum_{i<|\{a, F a, \ldots\}|} k_{i} F^{i} a: \text { each } k_{i}<|a|\right\}
$$

would be finite, and $\Gamma=\mathbb{Z}[F] \Sigma$ would be finite, a contradiction.

Fix $a \in \Sigma$ of infinite $F$-orbit. Fix $s$ such that $C^{s-1}>D E$.

Claim 5.14. Suppose $\sigma \in\{-a, 0, a\}^{*} \backslash\{0\}^{*}$. Then $[\sigma]_{F^{s}} \neq 0$.

Proof. We show that $\lambda_{\Sigma}\left([\sigma]_{F^{s}}\right) \neq 0$, which will suffice.

Let $\ell=|\sigma|>0$. We may assume $\sigma$ has no trailing zeroes and, by possibly negating, that $\sigma_{\ell-1}=a$; so

$$
\lambda_{\Sigma}\left([\sigma]_{F^{s}}\right)=\lambda_{\Sigma}\left(\left[\sigma_{0} \cdots \sigma_{\ell-2}\right]_{F^{s}}+F^{s(\ell-1)} a\right)
$$

We look to apply the reverse ultrametric inequality. Note that $\lambda_{\Sigma}\left(\left[\sigma_{0} \cdots \sigma_{\ell-2}\right]_{F^{s}}\right) \leq 2^{s(\ell-2)+1}=$ $C^{s(\ell-2)+1}$ by definition of $\lambda_{\Sigma}$. Thus $\lambda_{\Sigma}\left(F^{s(\ell-1)} a\right) \geq C^{s(\ell-1)} E^{-1}>C^{s(\ell-2)+1} D \geq D \lambda_{\Sigma}\left(\left[\sigma_{0} \cdots \sigma_{\ell-2}\right]_{F s}\right)$ by canonicity and since $C^{s-1}>D E$; note that canonicity applies since $a$ has infinite $F$-orbit. So by the reverse ultrametric inequality

$$
\lambda_{\Sigma}\left([\sigma]_{F^{s}}\right) \geq D^{-1} \lambda_{\Sigma}\left(F^{s(\ell-1)} a\right) \geq D^{-1} E^{-1} C^{s(\ell-1)}>0
$$

So in particular $[\sigma]_{F s} \neq 0$.

Claim 5.14 
It follows that $[\cdot]_{F^{s}}\left\lceil\{0, a\}^{*} a\right.$ is injective. Indeed, suppose $\sigma, \tau \in\{0, a\}^{*} a$ are distinct; then we can write $[\sigma]_{F^{s}}-[\tau]_{F^{s}}=[\nu]_{F^{s}}$ for some $\nu \in\{-a, 0, a\}^{*} \backslash\{0\}^{*}$. Hence by the claim $[\sigma]_{F^{s}}-[\tau]_{F^{s}} \neq 0$.

But then

$$
f_{\Gamma, \lambda_{\Sigma}}\left(2^{s \ell+1}\right)=\left|\left\{[\sigma]_{F}: \sigma \in \Sigma^{*},|\sigma| \leq s \ell+1\right\}\right| \geq\left|\left\{[\sigma]_{F^{s}}: \sigma \in\{0, a\}^{(\ell)} a\right\}\right|=\left|\{0, a\}^{(\ell)} a\right|=2^{\ell}
$$

So $f_{\Gamma, \lambda_{\Sigma}} \notin O\left(\log (x)^{d}\right)$ for any $d$; so $\Gamma$ is not $\lambda_{\Sigma}$-sparse, and hence by Theorem $5.9 \Gamma$ is not $F$-sparse.

Suppose now that $H$ is an arbitrary infinite $F$-invariant subgroup of $\Gamma$. By Corollary 3.10 there is an $F^{s}$-spanning set for $H$ for some $s>0$; so by the case $H=\Gamma$ we get that $H$ isn't $F$-sparse in $H$. So Corollary 5.12 yields that $H$ isn't $F$-sparse in $\Gamma$.

2. Again using Proposition 5.4 (1) we may assume there is a length function $\lambda$ for $(\Gamma, F)$. Since $F$-sparsity is closed under translation (Proposition $5.4(2)$ ) we may assume $A$ contains an infinite $H \leq \Gamma$. Since $\Gamma$ is finitely generated and $H$ is infinite there is some $a \in H$ of infinite order; we will show that $\mathbb{Z} a$ isn't $\lambda$-sparse. We show by induction on $k$ that if $1 \leq n \leq 2^{k}$ then $\lambda(n a) \leq D^{k-1} \lambda(a)$. The base case is immediate. For the induction step, suppose the claim holds of $k$; suppose $2^{k}<n \leq 2^{k+1}$. Then

$$
\lambda(n a)=\lambda\left(2^{k} a+\left(n-2^{k}\right) a\right) \leq D \max \left(2^{k} a,\left(n-2^{k}\right) a\right) \leq D \max \left(D^{k-1} \lambda(a), D^{k-1} \lambda(a)\right) \leq D^{k} \lambda(a)
$$

by the induction hypothesis.

Then $\left\{n a: n \in \mathbb{Z}, \lambda(n a) \leq D^{k-1} \lambda(a)\right\} \supseteq\left\{n a: 1 \leq n \leq 2^{k}\right\}$ contains at least $2^{k}$ elements. So $f_{\mathbb{Z} a, \lambda}(x) \notin O\left(\log (x)^{d}\right)$ for any $d$, and $\mathbb{Z} a$ isn't $\lambda$-sparse. But $A \supseteq H \supseteq \mathbb{Z} a$; so $f_{A, \lambda}(x) \geq f_{\mathbb{Z} a, \lambda}(x)$, and $A$ isn't $\lambda$-sparse. So $A$ isn't $F$-sparse by Theorem 5.9.

$\square$ Corollary 5.13

\section{$6 \quad F$-sparse sets and stable expansions of finitely generated abelian groups}

Fix an abelian group $\Gamma$ and a subset $A \subseteq \Gamma$. We say that $A$ is stable in $\Gamma$ if there do not exist arbitrarily long tuples $\left(a_{1}, \ldots, a_{N} ; b_{1}, \ldots, b_{N}\right)$ of elements of $\Gamma$ such that $a_{i}+b_{j} \in A$ if and only if $i \leq j$. This can be expressed model-theoretically by requiring that in $\operatorname{Th}(\Gamma, 0,+, A)$ the formula $x+y \in A$ be a stable formula. The question of which subsets of $\Gamma$ are stable has been of significant interest to model theorists for some time. We refine the question here as follows:

Question 6.1. Suppose $\Gamma$ is a finitely generated abelian group and $F: \Gamma \rightarrow \Gamma$ is an injective endomorphism, such that $(\Gamma, F)$ admits a spanning set for some $r>0$. Which $F$-automatic sets of $\Gamma$ are stable?

In [5] I answered this question in the classical case where $\Gamma=\mathbb{Z}$ and $F$ is multiplication by a positive integer. Here, we generalize the methods of [5] to answer the above question for $F$-sparse sets. This is Theorem 6.3 below. There are some issues that arise in generalizing; we focus our exposition on what needs to be done beyond what was done in [5].

Our answer will be in terms of the " $F$-cycles" introduced in $[6]$ :

Definition 6.2. Suppose $\Gamma$ is an abelian group and $F$ is an injective endomorphism. An $F$-cycle is a set of the form $C\left(a ; F^{\delta}\right):=\left\{a+F^{\delta} a+\cdots+F^{\delta n} a: n<\omega\right\}$. An $F$-set of $\Gamma^{m}$ is a finite union of sets of the form $\gamma+H+C\left(a_{1} ; F^{r_{1}}\right)+\cdots+C\left(a_{n} ; F^{r_{n}}\right)$ where $\gamma \in \Gamma^{m}, H \leq \Gamma^{m}$ is $F$-invariant, each $a_{i} \in \Gamma^{m}$, and each $r_{i}>0$. A groupless $F$-set is a finite union of sets of the form $\gamma+C\left(a_{1} ; F^{r_{1}}\right)+\cdots+C\left(a_{n} ; F^{r_{n}}\right)$ with $\gamma, a_{i}, r_{i}$ as above. The $F$-structure $(\Gamma, \mathcal{F})$ on $\Gamma$ has domain $\Gamma$ and a predicate for every $F$-set of every $\Gamma^{m}$.

Note in particular that the graph of addition is an $F$-invariant subgroup of $\Gamma^{3}$, and is thus an $F$-set; so $(\Gamma, \mathcal{F})$ expands $(\Gamma,+)$.

In $[6]$ it was shown that if $\Gamma$ is finitely generated and

$$
\bigcap_{i \in \mathbb{N}}\left(F^{i}\right)=\{0\}
$$

holds in $\mathbb{Z}[F]$ then the $F$-structure $(\Gamma, \mathcal{F})$ is stable. In particular, all $F$-sets are stable in $\Gamma$. Here we prove a converse for $F$-sparse sets. 
Theorem 6.3. Suppose $\Gamma$ is a finitely generated abelian group and $F$ is an injective endomorphism of $\Gamma$ such that $\Gamma$ admits an $F^{r}$-spanning set for some $r>0$. If $A \subseteq \Gamma$ is $F$-sparse and stable in $\Gamma$ then $A$ is a finite Boolean combination of groupless F-sets.

We would like to use the results of [6] to deduce quantifier elimination of $(\Gamma, \mathcal{F})$; unfortunately, this requires that $\mathbb{Z}[F]$ satisfy $(\dagger)$, which doesn't follow from the existence of a spanning set. Consider for example $\Gamma=\mathbb{Z} \times(\mathbb{Z} / 2 \mathbb{Z})$ with the endomorphism $F(a, b)=(2 a, b)$. One can check using e.g. Theorem 3.11 that $\Gamma$ admits an $F^{r}$-spanning set for some $r>0$, but $F-2=F^{i}(F-2) \in\left(F^{i}\right)$ for all $i \in \mathbb{N}$.

We give a sufficient condition for $(\dagger)$ to hold, and then reduce Theorem 6.3 to the case where this condition holds.

Lemma 6.4. If $\Gamma$ is torsion-free and admits an $F^{r}$-spanning set for some $r>0$ then $\mathbb{Z}[F]$ satisfies ( $\dagger$ ).

Proof. Since $\bigcap_{i \in \mathbb{N}}\left(F^{i}\right)=\bigcap_{i \in \mathbb{N}}\left(F^{r i}\right)$, we may assume there is a length function $\lambda$ for $(\Gamma, F)$ (possibly replacing $F$ by a power thereof) with associated constants $C, D, E$. By Lemma 3.5 and Remark 3.6 we may assume canonicity applies to all non-zero elements.

Suppose $G \in \bigcap_{i \in \mathbb{N}}\left(F^{i}\right)$, and suppose $a \in \Gamma$; suppose for contradiction that $G a \neq 0$. Let $t \in \mathbb{R}$ be the smallest non-zero value in $\lambda(\Gamma)$ (which must exist by the Northcott property). For $n \in \mathbb{N}$ since $G \in\left(F^{n}\right.$ ) there is $G_{n} \in \mathbb{Z}[F]$ such that $G=F^{n} G_{n}$; since $G a \neq 0$ we get that $G_{n} a \neq 0$. Then $\lambda(G a)=\lambda\left(F^{n} G_{n} a\right) \geq$ $C^{n} E^{-1} \lambda\left(G_{n} a\right) \geq C^{n} E^{-1} t$. (Note that $\lambda\left(G_{n} a\right) \neq 0$ else canonicity would contradict Northcott.) But this grows without bound, a contradiction. So $G a=0$ for all $a \in \Gamma$, and $G=0$.

We can now show Theorem 6.3 under the assumption that $\Gamma$ is torsion-free.

Proposition 6.5. Suppose $\Gamma$ is torsion-free and admits an $F^{r}$-spanning set for some $r>0$. If $A \subseteq \Gamma$ is stable and $F$-sparse then $A$ is a finite Boolean combination of groupless F-sets.

Proof. By replacing $F$ with a power thereof we may assume $\Gamma$ admits an $F$-spanning set (since $A$ is also $F^{r}$-sparse by Proposition $\left.5.4(1)\right)$. By Proposition 5.3 we can write $A$ as a finite union of sets of the form $\gamma+\left[a_{1}^{*} \cdots a_{n}^{*}\right]_{F}$ for some $s>0$ and $\gamma, a_{1}, \ldots, a_{n} \in \Gamma$. If we let $b_{1}, \ldots, b_{n} \in \Gamma$ be such that $a_{i}=\sum_{j \geq i} b_{i}$ then we can rewrite

$$
\gamma+\left[a_{1}^{*} \cdots a_{n}^{*}\right]_{F^{s}}=\gamma+\left\{\left[b_{1}^{e_{1}}\right]_{F^{s}}+\cdots+\left[b_{n}^{e_{n}}\right]_{F^{s}}: e_{1} \leq \cdots \leq e_{n}\right\}
$$

and thus write $A$ as a finite union of sets of this form. Fix one such $\gamma+\left\{\left[b_{1}^{e_{1}}\right]_{F^{s}}+\cdots+\left[b_{n}^{e_{n}}\right]_{F^{s}}: e_{1} \leq \cdots \leq e_{n}\right\}$ in the union; we show it is contained in some $B$ of the desired form that is itself contained in $A$.

We will proceed by examining the $e_{i}$ such that $\left[b_{1}^{e_{1}}\right]_{F^{s}}+\cdots+\left[b_{n}^{e_{n}}\right]_{F^{s}} \in A$.

Claim 6.6. $X:=\left\{\left(e_{1}, \ldots, e_{n}\right) \in \mathbb{N}^{n}:\left[b_{1}^{e_{1}}\right]_{F^{s}}+\cdots+\left[b_{n}^{e_{n}}\right]_{F^{s}} \in A-\gamma\right\}$ is quantifier-free definable in $(\mathbb{N}, 0, S, \delta \mathbb{N})$ for some $\delta \in \mathbb{N} \backslash\{0\}$, where $S$ is the successor function.

Proof. Fix $\sigma \in S_{n}$, and suppose $e_{\sigma(1)} \leq \cdots \leq e_{\sigma(n)}$. Let $c_{i}=\sum_{j \geq i} b_{\sigma(i)}$; so

$$
\left[b_{1}^{e_{1}}\right]_{F^{s}}+\cdots+\left[b_{n}^{e_{n}}\right]_{F^{s}} \in A \Longleftrightarrow\left[c_{1}^{e_{1}} c_{2}^{e_{2}-e_{1}} \cdots c_{n}^{e_{n}-e_{n-1}}\right]_{F^{s}} \in A-\gamma
$$

By [2, Lemmas 5.6 and 5.7] there is an $F^{s}$-spanning set $\Sigma$ containing all the $c_{i}$; so by Proposition 2.6 $\left\{\sigma \in \Sigma^{*}:[\sigma]_{F^{s}} \in A-\gamma\right\}$ is regular. Then as argued in the proof of [5, Proposition 2.2] there is some Boolean combination $\varphi\left(x_{1}, \ldots, x_{n}\right)$ of congruences and equalities between one variable and one constant such that

$$
\left(e_{1}, \ldots, e_{n}\right) \in X \Longleftrightarrow\left[c_{1}^{e_{1}} c_{2}^{e_{2}-e_{1}} \cdots c_{n}^{e_{n}-e_{n-1}}\right]_{F^{s}} \Longleftrightarrow(\mathbb{N}, 0, S, \delta \mathbb{N}) \models \varphi\left(e_{1}, e_{2}-e_{1}, \ldots, e_{n}-e_{n-1}\right)
$$

(again, under the assumption that $\left.e_{\sigma(1)} \leq \cdots \leq e_{\sigma(n)}\right)$. But a congruence or equality between a constant $N \in \mathbb{N}$ and either $e_{1}$ or $e_{i+1}-e_{i}$ can be expressed as a formula in $(\mathbb{N}, 0, S, \delta \mathbb{N})$, for some sufficiently large $\delta$. So this is in turn equivalent to $(\mathbb{N}, 0, S, \delta \mathbb{N}) \models \psi\left(e_{1}, \ldots, e_{n}\right)$ for some quantifier-free $\psi$.

Doing this for all $\sigma$ and taking LCMs of the $\delta$ and disjunctions over the possible orderings of the $e_{i}$, we find that there is a single quantifier-free formula $\varphi$ in $(\mathbb{N}, 0, S, \delta \mathbb{N},<)$ such that $X=\varphi\left(\mathbb{N}^{n}\right)$. Then $\varphi$ is a 
stable formula under any partitioning of its variables: large ladders for $\varphi$ would yield large ladders for the corresponding partitioning of

$$
\bigwedge_{i=1}^{n}\left(x_{i} \in\left[b_{i}^{*}\right]_{F s}\right) \wedge\left(x_{1}+\cdots+x_{n} \in A-\gamma\right)
$$

and the latter is stable under any partitioning of the variables as $A$ is stable (and addition is commutative and associative).

But the stable quantifier-free formulas in $(\mathbb{N}, 0, S, \delta \mathbb{N},<)$ are known to be quantifier-free formulas in $(\mathbb{N}, 0, S, \delta \mathbb{N})$; this is [5, Proposition 3.3]. So $X$ is quantifier-free definable in $(\mathbb{N}, 0, S, \delta \mathbb{N})$.

$\square$ Claim 6.6

Claim 6.7. $Y:=\left\{\left[b_{1}^{e_{1}}\right]_{F^{s}}+\cdots+\left[b_{n}^{e_{n}}\right]_{F^{s}}:\left(e_{1}, \ldots, e_{n}\right) \in X\right\}$ is definable in $(\Gamma, \mathcal{F})$.

Proof. Fix $a \in \Gamma$ such that $F^{i} a \neq F^{j} a$ for $i \neq j$; canonicity of any length function for any $\left(\Gamma, F^{t}\right)$ shows such $a$ must exist. Then $\left[a^{i}\right]_{F^{s}} \neq\left[a^{j}\right]_{F^{s}}$ for $i \neq j$; indeed, otherwise applying $F^{s}-1$ to both sides we would have $F^{s i} a=F^{s j} a$, a contradiction.

Consider $\Phi: \mathbb{N} \rightarrow \Gamma$ given by $i \mapsto\left[a^{i}\right]_{F s}$. By a similar argument to the one given in the proof of [5, Lemma 3.7] we get that $\Phi$ is an interpretation of $(\mathbb{N}, 0, S, \delta \mathbb{N})$ in $(\Gamma, \mathcal{F})$. Furthermore each map $\left[a^{i}\right]_{F^{s}} \mapsto\left[b^{i}\right]_{F^{s}}$ is definable in $(\Gamma, \mathcal{F})$, as is addition. So $Y=\left\{\left[b_{1}^{e_{1}}\right]_{F^{s}}+\cdots+\left[b_{n}^{e_{n}}\right]_{F^{s}}:\left(\left[a^{e_{1}}\right]_{F^{s}}, \ldots,\left[a^{e_{n}}\right]_{F^{s}}\right) \in \Phi(X)\right\}$ is definable in $(\Gamma, \mathcal{F})$, as desired.

$\square$ Claim 6.7

We get by Lemma 6.4 that $(\Gamma, F)$ satisfies $(\dagger)$; note further since $\Gamma$ is an infinite finitely generated abelian group that $\mathbb{Z}$ embeds into $\mathbb{Z}[F]$. So by $[6$, Theorem $\mathrm{A}](\Gamma, \mathcal{F})$ admits quantifier elimination; so $Y$ (and hence $\gamma+Y)$ is a Boolean combination of $F$-sets. At this point the argument given in the proof of [5, Theorem 3.1] shows that $\gamma+Y$ is a Boolean combination of $F$-sparse $F$-sets. Now by Corollary 5.13 an $F$-sparse $F$-set must be groupless; so $\gamma+Y$ is a Boolean combination of groupless $F$-sets. But

$$
\gamma+\left\{\left[b_{1}^{e_{1}}\right]_{F^{s}}+\cdots+\left[b_{n}^{e_{n}}\right]_{F^{s}}: e_{1} \leq \cdots \leq e_{n}\right\} \subseteq \gamma+Y \subseteq A
$$

So we can replace $\gamma+\left\{\left[b_{1}^{e_{1}}\right]_{F^{s}}+\cdots+\left[b_{n}^{e_{n}}\right]_{F^{s}}: e_{1} \leq \cdots \leq e_{n}\right\}$ with $\gamma+Y$ in the union defining $A$ without changing the union. Applying this to all sets in the union, we have written $A$ as a Boolean combination of groupless $F$-sets.

$\square$ Proposition 6.5

Proof of Theorem 6.3. By Proposition 5.4 (1) we may replace $F$ with $F^{r}$, and thus assume that $\Gamma$ admits an $F$-spanning set $\Sigma$. Using the fundamental theorem of finitely generated abelian groups we can write $\Gamma=\Gamma_{0} \oplus H$ where $H$ is the torsion subgroup of $\Gamma$ and $\Gamma_{0}$ is torsion-free. Let $F_{0}: \Gamma_{0} \rightarrow \Gamma_{0}$ be $\left(\pi_{0} \circ F\right) \uparrow \Gamma_{0}$, where $\pi_{0}: \Gamma \rightarrow \Gamma_{0}$ is the projection. Using the fact that $H$ is $F$-invariant one can show that $\pi_{0}(\Sigma)$ is an $F_{0}^{r}$-spanning set for $\Gamma_{0}$. One can further check that if $v_{i}, w_{i} \in \Sigma^{*}$ then $\pi_{0}\left(\left[v_{0} w_{1}^{k_{1}} v_{1} \cdots w_{n}^{k_{n}} v_{n}\right]_{F^{r}}\right)=$ $\left[\pi_{0}\left(v_{0}\right) \pi_{0}\left(w_{1}\right)^{k_{1}} \pi_{0}\left(v_{1}\right) \cdots \pi_{0}\left(w_{n}\right)^{k_{n}} \pi_{0}\left(w_{n}\right)\right]_{F_{0}^{r}}$, where for $\sigma \in \Sigma^{*}$ we obtain $\pi_{0}(\sigma) \in\left(\pi_{0}(\Sigma)\right)^{*}$ by applying $\pi_{0}$ to each letter of $\sigma$. So if $B \subseteq \Gamma$ is $F$-sparse then $\pi_{0}(B) \subseteq \Gamma_{0}$ is $F_{0}$-sparse.

For $b \in H$ let $A_{b}=\left\{a \in \Gamma_{0}: a \oplus b \in A\right\}$; note that $x+y \in A_{b}$ is stable in $\Gamma_{0}$. We wish to show that $A_{b}$ is $F$-automatic in $\Gamma$; since regular languages are closed under intersection and $A-b$ is $F$-automatic it suffices to show that $\Gamma_{0}$ is. Fix a generating set $\left\{\gamma_{1}, \ldots, \gamma_{\ell}\right\}$ for $\Gamma$, and write

$$
F \gamma_{i}=\sum_{j=1}^{\ell} a_{i j} \gamma_{j}
$$

for $a_{i j} \in \mathbb{Z}$; let $X=\left(a_{i j}+|H| \mathbb{Z}\right)_{i, j=1}^{\ell} \in M_{\ell}(\mathbb{Z} /|H| \mathbb{Z})$. We construct a DFA recognizing $\Gamma_{0}$. Let $\pi_{H}: \Gamma \rightarrow H$ be the projection. The idea is to observe that if $c=c_{1} \gamma_{1}+\cdots+c_{\ell} \gamma_{\ell} \in \Gamma$ then to compute $\pi_{H}\left(F^{n} c\right)$ it suffices to know $X^{n}\left(c_{i}+|H| \mathbb{Z}\right)_{i=1}^{\ell}$, and since $X$ is a matrix over a finite ring, its powers can be tracked using a finite automaton. Using this we can determine $\pi_{H}\left([\sigma c]_{F}\right)$ from $\pi_{H}\left([\sigma]_{F}\right)$ and $X^{n}\left(c_{i}+|H| \mathbb{Z}\right)_{i=1}^{\ell}$, which are finitary objects.

The set of states is $Q=\left\{(h, Y): h \in H, Y \in M_{\ell}(\mathbb{Z} /|H| \mathbb{Z})\right\}$. The initial state is $q_{0}=(0, I)$, and the finish states are $\Omega=\left\{(0, Y): Y \in M_{\ell}(\mathbb{Z} /|H| \mathbb{Z})\right\}$. For the transition map, suppose we are in state $(h, Y)$ and receive 
input $c \in \Sigma$. Let $Y^{\prime}=X Y$. Write $c=c_{1} \gamma_{1}+\cdots+c_{\ell} \gamma_{\ell}$ for $c_{i} \in \mathbb{Z}$, and let $v=\left(c_{i}+|H| \mathbb{Z}\right)_{i=1}^{\ell} \in(\mathbb{Z} /|H| \mathbb{Z})^{\ell}$. Let

$$
h^{\prime}=h+\sum_{i=1}^{\ell} \pi_{H}\left((Y v)_{i}\right)
$$

where $(Y v)_{i} \in \mathbb{Z} /|H| \mathbb{Z}$ is the $i^{\text {th }}$ entry of $Y v$. Note that this is well-defined since $\pi_{H}(|H| \Gamma)=0$. Our machine then transitions to $\left(h^{\prime}, Y^{\prime}\right)$. One can check by induction on $|\sigma|$ that $\delta\left(q_{0}, \sigma\right)=\left(\pi_{H}\left([\sigma]_{F}\right), X^{|\sigma|}\right)$. So our machine recognizes $\pi_{H}^{-1}(0)=\Gamma_{0}$, and $\Gamma_{0}$ (and hence $\left.A_{b}\right)$ is $F$-automatic in $\Gamma$.

Furthermore $A_{b} \subseteq A-b$, and $A$ is $F$-sparse; so by Proposition 5.4 (2) and (4) $A_{b}$ is $F$-sparse in $\Gamma$. So as remarked above we get that $A_{b}$ is $F_{0}$-sparse in $\Gamma_{0}$. So by Proposition 6.5 each $A_{b}$ is a Boolean combination of groupless $F_{0}$-sets. So since

$$
A=\bigcup_{b \in H}\left(A_{b}+b\right)
$$

it suffices to show that we can write $C\left(a ; F_{0}^{s}\right)$ as a union of translates of sets of the form $C\left(b ; F^{t}\right)$. Let $\sigma=a 0^{s-1} \in \Gamma^{*}$. Using the above automaton, we see that $\delta\left(q_{0},\left[\sigma^{i}\right]_{F}\right)$, and hence $\pi_{H}\left(\left[\sigma^{i}\right]_{F}\right)$, is ultimately periodic in $i$; say $\pi_{H}\left(\left[\sigma^{i+\mu}\right]_{F}\right)=\pi_{H}\left(\left[\sigma^{i}\right]_{F}\right)$ for $i \geq N$. Then

$$
C\left(a ; F_{0}^{s}\right)=\left\{\left[\sigma^{i}\right]_{F_{0}}: i>0\right\}=\left\{\left[\sigma^{i}\right]_{F_{0}}: 0<i<N\right\} \cup \bigcup_{j<\mu}\left\{\left[\sigma^{N+j+i \mu}\right]_{F_{0}}: i \in \mathbb{N}\right\}
$$

But $\left[\sigma^{N+j+i \mu}\right]_{F}=\pi_{H}\left(\left[\sigma^{N+j+i \mu}\right]_{F}\right)+\left[\sigma^{N+j+i \mu}\right]_{F_{0}}=\pi_{H}\left(\left[\sigma^{N+j}\right]_{F}\right)+\left[\sigma^{N+j+i \mu}\right]_{F_{0}}$. So

$$
\begin{aligned}
\left\{\left[\sigma^{N+j+i \mu}\right]_{F_{0}}: i \in \mathbb{N}\right\} & =\pi_{H}\left(\left[\sigma^{N+j}\right]_{F}\right)+\left\{\left[\sigma^{N+j+i \mu}\right]_{F}: i \in \mathbb{N}\right\} \\
& =\pi_{H}\left(\left[\sigma^{N+j}\right]_{F}\right)+\left[\sigma^{N+j}\right]_{F}+C\left(F^{(N+j) s}\left[\sigma^{\mu}\right]_{F} ; F^{\mu}\right)
\end{aligned}
$$

Substituting this into the above expression for $C\left(a ; F_{0}^{s}\right)$, we have written $C\left(a ; F_{0}^{s}\right)$ as a union of translates of $C\left(b ; F^{\mu}\right)$, as desired.

$\square$ Theorem 6.3

It is worth pointing out that, assuming the existence of spanning sets, there are always $F$-sparse sets that aren't $F$-sets. Indeed, fix any $a \in \Gamma$ of infinite $F$-orbit. One can check that $A=\left\{\left(\begin{array}{l}F^{i} a \\ F^{j} a\end{array}\right): i \leq j\right\} \subseteq \Gamma^{2}$ is $F$-sparse. If $A$ were an $F$-set then by [6] it would be stable, contradicting the fact that $\left(\begin{array}{c}F^{i} a \\ 0\end{array}\right)+\left(\begin{array}{c}0 \\ F^{j} a\end{array}\right) \in A$ if and only if $i \leq j$. With some effort one can encode sets like this into subsets of $\Gamma$ itself.

\section{$7 \quad$ NIP expansions of $(\Gamma,+)$}

We now turn our attention to NIP expansions of $(\Gamma,+)$. We produce a class of subsets $A \subseteq \Gamma$, which we call the $F$-EDP sets, that contains the $F$-sparse sets; we show that if $A$ is $F$-EDP then $(\Gamma,+, A)$ is NIP. Our argument generalizes that of [5, Subsection 6.2], which deals with the case when $\Gamma=\mathbb{Z}$ and $F$ is multiplication by some $d>1$. As an application of our general result, we will see that $\left(\mathbb{F}_{p}[t],+, \times\left\lceil t^{\mathbb{N}}\right)\right.$ is NIP, where $\times \mid t^{\mathbb{N}}$ is the graph of multiplication on $t^{\mathbb{N}}$.

We introduce some convenient multi-index notation. Suppose $\mathbf{s}=\left(\sigma_{1}, \ldots, \sigma_{n}\right)$ with $\sigma_{1}, \ldots, \sigma_{n} \in \Gamma^{*}$ and $\mathbf{k}=\left(k_{1}, \ldots, k_{n}\right) \in \mathbb{N}^{n}$. Then by $\mathbf{s}^{\mathbf{k}}$ we mean the string $\sigma_{1}^{k_{1}} \cdots \sigma_{n}^{k_{n}}$. Note that if $\mathbf{0}=(0, \ldots, 0)$ then $\mathbf{s}^{\mathbf{0}}=\varepsilon$, the empty word.

Definition 7.1. By an $F$-exponentially definable in Presburger ( $F$-EDP) subset of $\Gamma$ we mean a set of the form $\left[\mathbf{s}^{\varphi(\mathbb{N})}\right]_{F}:=\left\{\left[\mathbf{s}^{\mathbf{k}}\right]_{F}:(\mathbb{N},+) \models \varphi(\mathbf{k})\right\}$ for some tuple $\mathbf{s}$ of strings over $\Gamma$ and some formula $\varphi(\mathbf{x})$ with $|\mathbf{x}|=|\mathbf{s}|$.

\section{Lemma 7.2.}

1. F-EDP sets are closed under finite union.

2. F-sparse sets are F-EDP. 
Proof.

1. It suffices to check pairwise unions. Suppose we are given $F$-EDP sets $\left[\mathbf{s}_{1}^{\varphi(\mathbb{N})}\right]_{F}$ and $\left[\mathbf{s}_{2}^{\psi(\mathbb{N})}\right]_{F}$. Then their union can be written as $\left[\left(\mathbf{s}_{1} \mathbf{s}_{2}\right)^{\chi(\mathbb{N})}\right]_{F}$ where $\chi(\mathbf{x}, \mathbf{y})$ is $(\varphi(\mathbf{x}) \wedge \mathbf{y}=\mathbf{0}) \vee(\psi(\mathbf{y}) \wedge \mathbf{x}=\mathbf{0})$, and is thus $F$-EDP.

2. Suppose we are given an $F^{r}$-spanning set $\Sigma$ for some $r>0$ and a sparse $L \subseteq \Sigma^{*}$. By Fact 5.2 we may assume $L$ is a finite union of simple sparse languages; by part (1) we may assume $L$ itself is simple sparse, say $L=v_{0} w_{1}^{*} v_{1} \cdots w_{n}^{*} v_{n}$ for $v_{i}, w_{i} \in \Sigma^{*}$. Given $\sigma=s_{0} \cdots s_{|\sigma|-1} \in \Sigma^{*}$ let $\sigma^{\prime}=$ $s_{0} 0^{r-1} s_{1} 0^{r-1} \cdots s_{|\sigma|-1} 0^{r-1}$. Then $(\sigma \tau)^{\prime}=\sigma^{\prime} \tau^{\prime}$ and $\left[\sigma^{\prime}\right]_{F}=[\sigma]_{F^{r}}$. So $[L]_{F^{r}}=\left\{\left[v_{0}^{\prime}\left(w_{1}^{\prime}\right)^{k_{1}} v_{1}^{\prime} \cdots\left(w_{n}^{\prime}\right)^{k_{n}} v_{n}^{\prime}\right]_{F}\right.$ : $\left.k_{1}, \ldots, k_{n} \in \mathbb{N}\right\}$ is $F$-EDP.

The $F$-EDP sets contain significantly more than just the $F$-sparse sets. For instance, if $a, b, c \in \Gamma$ then $\left\{\left[a^{i} b^{i}\right]_{F}: i \in \mathbb{N}\right\}$ and $\left\{\left[a^{i} b^{j} c^{i+j}\right]_{F}: i, j \in \mathbb{N}\right\}$ are $F$-EDP, but are not typically $F$-automatic, and hence not $F$-sparse.

Our goal is to prove that if we start with a weakly minimal abelian group $(\Gamma,+)$, and if $A \subseteq \Gamma$ is $F$-EDP, then $(\Gamma,+, A)$ is an NIP structure. Recall that $\Gamma$ being weakly minimal means that $\operatorname{Th}(\Gamma,+)$ is superstable and of $U$-rank 1. Equivalently, for all $n>0, n \Gamma$ and the subgroup of $n$-torsion are either finite or of finite index in $\Gamma$-see for example [4, Proposition 3.1]. So, for example, all finitely generated abelian groups are weakly minimal.

We begin by adapting Proposition 5.3 to EDP sets.

Lemma 7.3. If $A$ is $F$-EDP then there is $s_{0}$ such that for all $s \in s_{0} \mathbb{N}$ we can write $A$ in the form $\left[\mathbf{a}^{\varphi(\mathbb{N})}\right]_{F^{s}}$ for some formula $\varphi$ in Presburger arithmetic and some tuple a of elements of $\Gamma$ (i.e. strings of length 1 ).

Proof. Write $A=\left\{\left[\mathbf{s}^{\mathbf{k}}\right]_{F}:(\mathbb{N},+) \models \varphi(\mathbf{k})\right\}$ where $\mathbf{s}=\left(\sigma_{1}, \ldots, \sigma_{n}\right)$. Let $s_{0}=\operatorname{lcm}\left(\left|\sigma_{1}\right|, \ldots,\left|\sigma_{n}\right|\right)$, and suppose $s \in s_{0} \mathbb{N}$. If $\sigma_{i}^{\prime}=\sigma_{i}^{\frac{s}{\sigma_{i}}}$ then

$$
A=\bigcup_{i_{1}<\frac{s}{\left|\sigma_{1}\right|}} \cdots \bigcup_{i_{n}<\frac{s}{\left|\sigma_{n}\right|}}\left\{\left[\left(\sigma_{1}^{\prime}\right)^{k_{1}^{\prime}} \sigma_{1}^{i_{1}} \cdots\left(\sigma_{n}^{\prime}\right)^{k_{n}^{\prime}} \sigma_{n}^{i_{n}}\right]_{F}:(\mathbb{N},+) \models \varphi\left(\frac{s}{\left|\sigma_{1}\right|} k_{1}^{\prime}+i_{1}, \ldots, \frac{s}{\left|\sigma_{n}\right|} k_{n}^{\prime}+i_{n}\right)\right\}
$$

Since sets of the desired form are closed under union (as in Lemma 7.2 (1)) it thus suffices to check the case

$$
A=\left\{\left[v_{0} w_{1}^{k_{1}} \cdots w_{n}^{k_{n}} v_{n}\right]_{F}:(\mathbb{N},+) \models \varphi\left(k_{1}, \ldots, k_{n}\right)\right\}
$$

where the $w_{i}$ all have the same length $s$. But by the argument given in [5, Lemma 3.5] (which generalizes to our context, as we noted in the proof of Proposition 5.3) there are $\gamma \in \Gamma$ and $\tau_{1}, \ldots, \tau_{n} \in \Gamma^{*}$ of length $s$ such that if each $k_{j}>0$ then we can write

$$
\left[v_{0} w_{1}^{k_{1}} \cdots w_{n}^{k_{n}} v_{n}\right]_{F}=\gamma+\left[\tau_{1}^{k_{1}-1} \cdots \tau_{n}^{k_{n}-1}\right]_{F}=\gamma+\left[a_{1}^{k_{1}-1} \cdots a_{n}^{k_{n}-1}\right]_{F^{s}}
$$

where $a_{i}=\left[\tau_{i}\right]_{F}$. The case where some $k_{j}=0$ can be dealt with inductively, again using closure under union; so it suffices to check the case $\gamma+\left\{\left[a_{1}^{k_{1}} \cdots a_{n}^{k_{n}}\right]_{F^{s}}:(\mathbb{N},+) \models \varphi\left(k_{1}, \ldots, k_{n}\right)\right\}$. But we can rewrite this as

$$
\bigcup_{i=1}^{n}\left\{\left[\left(\gamma+a_{i}\right) a_{i}^{k_{i}} \cdots a_{n}^{k_{n}}\right]_{F^{s}}:(\mathbb{N},+) \models \varphi\left(0, \ldots, 0, k_{i}+1, k_{i+1}, \ldots, k_{n}\right)\right\}
$$

which takes the desired form by closure under union.

Lemma 7.3

The source of NIP in Theorem 7.7 will be that $(\mathbb{N},+)$ is NIP. The following lemma and corollary relate automatic and $F$-EDP sets to sets definable in $(\mathbb{N},+)$.

Lemma 7.4. Suppose $\Lambda$ is a finite alphabet and $L \subseteq\left(\Lambda^{m}\right)^{*}$ is regular. Suppose $\mathbf{a}_{1}, \ldots, \mathbf{a}_{m}$ are tuples from $\Lambda$. Then the relation

$$
\left\{\left(\mathbf{k}_{1}, \ldots, \mathbf{k}_{m}\right):\left|\mathbf{a}_{1}^{\mathbf{k}_{1}}\right|=\cdots=\left|\mathbf{a}_{m}^{\mathbf{k}_{m}}\right|,\left(\begin{array}{c}
\mathbf{a}_{1}^{\mathbf{k}_{1}} \\
\vdots \\
\mathbf{a}_{m}^{\mathbf{k}_{m}}
\end{array}\right) \in L\right\} \subseteq \mathbb{N}^{\left|\mathbf{a}_{1}\right|} \times \cdots \times \mathbb{N}^{\left|\mathbf{a}_{m}\right|}
$$

is definable in $(\mathbb{N},+)$. (Here we identify $\left(\Lambda^{m}\right)^{*}$ with the subset of $\left(\Lambda^{*}\right)^{m}$ of tuples whose constituent strings all have the same length.) 
Proof. Fix an automaton $\left(\Lambda^{m}, Q, q_{0}, \Omega, \delta\right)$ for $L$; we show that for any $q_{1}, q_{2} \in Q$ the relation

$$
\left\{\left(\mathbf{k}_{1}, \ldots, \mathbf{k}_{m}\right):\left|\mathbf{a}_{1}^{\mathbf{k}_{1}}\right|=\cdots=\left|\mathbf{a}_{m}^{\mathbf{k}_{m}}\right|, \delta\left(q_{1},\left(\begin{array}{c}
\mathbf{a}_{1}^{\mathbf{k}_{1}} \\
\vdots \\
\mathbf{a}_{m}^{\mathbf{k}_{m}}
\end{array}\right)\right)=q_{2}\right\}
$$

is definable in $(\mathbb{N},+)$. Applying this to $q_{0}$ and the elements of $\Omega$ yields the desired result.

We apply induction on $\left|\mathbf{a}_{1}\right| \cdots\left|\mathbf{a}_{m}\right|$. For the base case, if some $\left|\mathbf{a}_{i}\right|=0$ then our relation is either empty or equivalent to all $\mathbf{k}_{i}$ being zero, depending on whether $q_{1}=q_{2}$, and both of these are definable in $(\mathbb{N},+)$.

For the induction step, suppose no $\left|\mathbf{a}_{i}\right|=0$. Write $\mathbf{a}_{i}=\left(a_{i 1}, \ldots, a_{i n_{i}}\right)$ and $\mathbf{k}_{i}=\left(k_{i 1}, \ldots, k_{i n_{i}}\right)$. Without loss of generality suppose $k_{11}$ is minimum among the $k_{i 1}$. If

$$
q=\delta\left(q_{1},\left(\begin{array}{c}
a_{11}^{k_{11}} \\
\vdots \\
a_{m 1}^{k_{11}}
\end{array}\right)\right)
$$

then our relation is equivalent to

$$
\left|a_{12}^{k_{12}} \cdots a_{1 n_{1}}^{k_{1 n_{1}}}\right|=\left|a_{21}^{k_{21}-k_{11}} a_{22}^{k_{22}} \cdots a_{2 n_{2}}^{k_{2 n_{2}}}\right|=\cdots=\left|a_{m 1}^{k_{m 1}-k_{11}} a_{m 2}^{k_{m 2}} \cdots a_{m n_{m}}^{k_{m n_{m}}}\right| \wedge \delta\left(q,\left(\begin{array}{c}
a_{12}^{k_{12}} \cdots a_{1 n_{1}}^{k_{1 n_{1}}} \\
a_{21}^{k_{21}-k_{11}} a_{22}^{k_{22}} \cdots a_{2 n_{2}}^{k_{2 n}} \\
\vdots \\
a_{m 1}^{k_{m 1}-k_{11}} a_{m 2}^{k_{m 2}} \cdots a_{m n_{m}}^{k_{m n_{m}}}
\end{array}\right)\right)=q_{2}
$$

which by the induction hypothesis is definable in $(\mathbb{N},+)$. Similarly we get definability in the case $k_{i 1}$ is minimum for some $i>1$. So taking disjunctions we get that our relation is definable in $(\mathbb{N},+) . \quad \square$ Lemma 7.4

Corollary 7.5. Suppose $\Gamma$ admits an F-spanning set. If $X \subseteq \Gamma^{m}$ is $F$-automatic and $\mathbf{a}_{1}, \ldots, \mathbf{a}_{m}$ are tuples from $\Gamma$ then

$$
\left\{\left(\mathbf{k}_{1}, \ldots, \mathbf{k}_{m}\right):\left(\begin{array}{c}
{\left[\mathbf{a}_{1}^{\mathbf{k}_{1}}\right]_{F}} \\
\vdots \\
{\left[\mathbf{a}_{m}^{\left.\mathbf{k}_{m}\right]_{F}}\right.}
\end{array}\right) \in X\right\} \subseteq \mathbb{N}^{\left|\mathbf{a}_{1}\right|} \times \cdots \times \mathbb{N}^{\left|\mathbf{a}_{m}\right|}
$$

is definable in $(\mathbb{N},+)$.

Proof. By [2, Lemma 5.6] there is an $F$-spanning set $\Sigma$ containing all the $a_{i j}$; one can then check that $\Sigma^{m}$ is an $F$-spanning set for $\Gamma^{m}$. Let $L \subseteq\left(\Sigma^{m}\right)^{*}$ be the set of representations of elements of $X$; so $L$ is regular. Then by previous lemma

$$
\left\{\left(\mathbf{k}_{1}, \ldots, \mathbf{k}_{m}, \ell_{1}, \ldots, \ell_{m}\right):\left|\mathbf{a}_{1}^{\mathbf{k}_{1}} 0^{\ell_{1}}\right|=\cdots=\left|\mathbf{a}_{m}^{\mathbf{k}_{m}} 0^{\ell_{m}}\right|,\left(\begin{array}{c}
\mathbf{a}_{1}^{\mathbf{k}_{1}} 0^{\ell_{1}} \\
\vdots \\
\mathbf{a}_{m}^{\mathbf{k}_{m}} 0^{\ell_{m}}
\end{array}\right) \in L\right\}
$$

is definable in $(\mathbb{N},+)$, say by $\varphi\left(\mathbf{k}_{1}, \ldots, \mathbf{k}_{m}, \ell_{1}, \ldots, \ell_{m}\right)$. Then $\exists \ell_{1} \ldots \exists \ell_{m} \varphi\left(\mathbf{k}_{1}, \ldots, \mathbf{k}_{m}, \ell_{1}, \ldots, \ell_{m}\right)$ defines the desired relation in $(\mathbb{N},+)$.

In order to apply the previous two results, we will need to relate the relations we wish to show are NIP to automatic sets. The following lemma does so. It is a straightforward generalization of a known result in classical automata theory; see for example [3, Theorem 6.1].

Lemma 7.6. Suppose $\Gamma$ admits an $F^{r}$-spanning set for some $r>0$. If $X \subseteq \Gamma^{m}$ is definable in $(\Gamma,+)$ with parameters from $\Gamma$ then $X$ is $F$-automatic. 
Proof. We apply structural induction on formulas. That the claim holds for $x=y$ and $x+y=z$ is $[2$, Lemma 6.7]; that the claim holds for a union or complement is because regular languages are closed under union and complementation. It remains to check existential quantification.

Suppose then that $X \subseteq \Gamma^{m+1}$ is $F$-automatic. Fix an $F^{r}$-spanning set $\Sigma$ for some $r>0$; then as before one can check that $\Sigma^{m+1}$ is an $F^{r}$-spanning set for $\Gamma^{m+1}$. So by Proposition 2.6 there is an automaton $M=\left(\Sigma^{m+1}, Q, q_{0}, \Omega, \delta\right)$ recognizing the representations over $\Sigma^{m+1}$ of elements of $X$. Consider the nondeterministic automaton $M^{\prime}=\left(\Sigma^{m}, Q, q_{0}, \Omega^{\prime}, \delta^{\prime}\right)$ where

- $\delta^{\prime}(q, \mathbf{a})=\left\{\delta\left(q,\left(\begin{array}{l}\mathbf{a} \\ b\end{array}\right)\right): b \in \Sigma\right\}$ for $\mathbf{a} \in \Sigma^{m}$, and

- $\Omega^{\prime}=\left\{q \in Q:\right.$ there is $\tau \in \Sigma^{*}$ such that $\left.\delta\left(q,\left(\begin{array}{c}\mathbf{0}^{|\tau|} \\ \tau\end{array}\right)\right) \in \Omega\right\}$.

Then if $\boldsymbol{\sigma} \in\left(\Sigma^{m}\right)^{*}$ is accepted by $M^{\prime}$ then there is $\tau_{1} \in \Sigma^{(|\boldsymbol{\sigma}|)}$ such that $\delta\left(q_{0},\left(\begin{array}{c}\boldsymbol{\sigma} \\ \tau_{1}\end{array}\right)\right) \in \Omega^{\prime}$; i.e. such that there is $\tau_{2} \in \Sigma^{*}$ such that $\delta\left(q_{0},\left(\begin{array}{c}\boldsymbol{\sigma} \mathbf{0}^{\left|\tau_{2}\right|} \\ \tau_{1} \tau_{2}\end{array}\right)\right) \in \Omega$. So $\left(\begin{array}{c}{[\boldsymbol{\sigma}]_{F^{r}}} \\ {\left[\tau_{1} \tau_{2}\right]_{F^{r}}}\end{array}\right) \in X$. Conversely suppose there is $b \in \Gamma$ such that $\left(\begin{array}{c}{[\boldsymbol{\sigma}]_{F^{r}}} \\ b\end{array}\right) \in X$; say $b=[\tau]_{F^{r}}$ for $\tau \in \Sigma^{*}$. Write $\tau=\tau_{1} \tau_{2}$ where $\left|\tau_{1}\right|=|\boldsymbol{\sigma}|$ (replacing $\tau$ with some $\tau 0^{k}$ if necessary). Then $\left(\begin{array}{c}{\left[\boldsymbol{\sigma} \mathbf{0}^{\left|\tau_{2}\right|}\right]_{F^{r}}} \\ {\left[\tau_{1} \tau_{2}\right]_{F^{r}}}\end{array}\right)=\left(\begin{array}{c}{[\boldsymbol{\sigma}]_{F^{r}}} \\ b\end{array}\right) \in X$; so $\left(\begin{array}{c}\boldsymbol{\sigma} \mathbf{0}^{\left|\tau_{2}\right|} \\ \tau_{1} \tau_{2}\end{array}\right)$ is accepted by $M$. So $\delta\left(q_{0},\left(\begin{array}{c}\boldsymbol{\sigma} \\ \tau_{1}\end{array}\right)\right) \in \delta^{\prime}\left(q_{0}, \boldsymbol{\sigma}\right) \cap \Omega^{\prime}$, and $M^{\prime}$ accepts $\boldsymbol{\sigma}$.

So $M^{\prime}$ recognizes the representations over $\Sigma^{r}$ of the projection of $X$ away from the last coordinate. So existentially quantifying an $F$-automatic set results in an $F$-automatic set.

$\square$ Lemma 7.6

Theorem 7.7. Suppose $(\Gamma,+)$ is a weakly minimal abelian group and admits an $F^{r}$-spanning set for some $r>0$; suppose $A \subseteq \Gamma$ is $F$-EDP. Then $(\Gamma,+, A)$ is NIP.

Proof. We apply [4, Theorem 2.9], which tells us that in a weakly minimal group $\Gamma$ if $A_{\Gamma}$ is NIP then so is $(\Gamma,+, A)$. Here $A_{\Gamma}$ is the induced structure of $(\Gamma,+)$ on $A$ : the domain of $A_{\Gamma}$ is $A$, and for every $(\Gamma,+)$ definable subset $X$ of $\Gamma^{m}$ with parameters from $\Gamma$ its trace $X \cap A^{m}$ on $A$ is an atomic relation of $A_{\Gamma}$. We show that $A_{\Gamma}$ is interpretible in $(\mathbb{N},+)$, and thus is NIP.

By Lemma 7.3 we may assume $A$ takes the form $\left[\mathbf{a}^{\varphi(\mathbb{N})}\right]_{F^{s}}$ for some $s \in r \mathbb{N}$. So $\Gamma$ admits an $F^{s}$-spanning set by [2, Lemma 5.7]. Let $\Phi: \mathbb{N}^{|\mathbf{a}|} \rightarrow \Gamma$ be $\mathbf{k} \mapsto\left[\mathbf{a}^{\mathbf{k}}\right]_{F s} ;$ so $\Phi$ is surjective $\varphi\left(\mathbb{N}^{|\mathbf{a}|}\right) \rightarrow A$. We show that $\Phi$ defines an interpretation of $A_{\Gamma}$ in $(\mathbb{N},+)$, and hence that $A_{\Gamma}$ is NIP.

By Corollary 7.5 the equivalence relation given by $\mathbf{k}_{1} \sim \mathbf{k}_{2}$ if and only if $\Phi\left(\mathbf{k}_{1}\right)=\Phi\left(\mathbf{k}_{2}\right)$ is definable in $(\mathbb{N},+)$ (since the diagonal in $\Gamma^{2 m}$ is $F$-automatic by [2, Lemma 6.7]). Suppose now that $X \subseteq \Gamma^{m}$ is definable with parameters in $(\Gamma, 0,+)$; it remains to show that $\varphi\left(\mathbb{N}^{|\mathbf{a}|}\right)^{m} \cap\left(\Phi^{m}\right)^{-1}(X)$ is definable in $(\mathbb{N},+)$. We get by Lemma 7.6 that $X$ is $F$-automatic; so by Corollary $7.5\left(\Phi^{m}\right)^{-1}(X)$ is definable in $(\mathbb{N},+)$. So $\varphi\left(\mathbb{N}^{|\mathbf{a}|}\right)^{m} \cap$ $\left(\Phi^{m}\right)^{-1}(X)$ is definable in $(\mathbb{N},+)$, and $\Phi$ defines an interpretation of $A_{\Gamma}$ in $(\mathbb{N},+)$.

$\square$ Theorem 7.7

As an application of Theorem 7.7, we prove the following:

Theorem 7.8. Suppose $p \geq 7$ is prime. Then $\left(\mathbb{F}_{p}[t],+, \times \mid t^{\mathbb{N}}\right)$ is NIP, where $\times \mid t^{\mathbb{N}}=\left\{\left(\begin{array}{c}t^{i} \\ t^{j} \\ t^{i+j}\end{array}\right): i, j \in \mathbb{N}\right\}$.

Proof. Let $F: \mathbb{F}_{p}[t] \rightarrow \mathbb{F}_{p}[t]$ be $f(t) \mapsto t f(t)$. Note that $\mathbb{F}_{p}$ is an $F$-spanning set for $\mathbb{F}_{p}[t]$. Indeed, an arbitrary element of $\mathbb{F}_{p}[t]$ is of the form $a_{0}+a_{1} t+\cdots+a_{n} t^{n}=\left[a_{0} a_{1} \cdots a_{n}\right]_{F}$, and the other axioms are easily verified using the facts that $\mathbb{F}_{p}$ is a subgroup and $\mathbb{F}_{p} \cap F\left(\mathbb{F}_{p}[t]\right)=\{0\}$. Furthermore $\left(\mathbb{F}_{p}[t],+\right)$ is weakly minimal: for each $n \in \mathbb{N}$ we have

$$
\begin{array}{r}
n \mathbb{F}_{p}[t]= \begin{cases}\mathbb{F}_{p}[t] & \text { if } p \nmid n \\
0 & \text { else }\end{cases} \\
\left\{a \in \mathbb{F}_{p}[t]: n a=0\right\}= \begin{cases}0 & \text { if } p \nmid n \\
\mathbb{F}_{p}[t] & \text { else }\end{cases}
\end{array}
$$


so weak minimality follows from $\left[4\right.$, Proposition 3.1]. So Theorem 7.7 applies, and $\left(\mathbb{F}_{p}[t],+, A\right)$ is NIP whenever $A \subseteq \mathbb{F}_{p}[t]$ is $F$-EDP.

We encode $\times\left\lceil t^{\mathbb{N}}\right.$ in an $F$-EDP subset of $\mathbb{F}_{p}[t]$. Write $\mathbb{F}_{p}=\mathbb{Z} / p \mathbb{Z}=\{\bar{n}: n \in \mathbb{Z}\}$. Let

$$
A=t^{\mathbb{N}} \cup \overline{2} t^{\mathbb{N}} \cup\left\{\overline{3} t^{i+j}-\overline{3} t^{i}-\overline{3} t^{j}: i, j \in \mathbb{N} \backslash\{0\}\right\}
$$

Note that

$$
\begin{aligned}
\left\{\overline{3} t^{i+j}-\overline{3} t^{i}-\overline{3} t^{j}: i, j \in \mathbb{N} \backslash\{0\}\right\}= & \left\{\left[(\overline{0})^{k}(\overline{-1})(\overline{0})^{\ell}(\overline{-1})(\overline{0})^{k-1} \overline{1}\right]_{F}: k, \ell \in \mathbb{N}, k>0\right\} \\
& \cup\left\{\left[(\overline{0})^{k}(\overline{-2})(\overline{0})^{k-1} \overline{1}\right]_{F}: k \in \mathbb{N} \backslash\{0\}\right\}
\end{aligned}
$$

is $F$-EDP as a union of $F$-EDP sets (see Lemma $7.2(1)$ ). Since $t^{\mathbb{N}}=\left\{\left[(\overline{0})^{k}\right]_{F}: k \in \mathbb{N}\right\}$, we get that $A$ itself is $F$-EDP as a union of $F$-EDP sets; so $\left(\mathbb{F}_{p}[t],+, A\right)$ is NIP. It remains to see that $\times\left\lceil t^{\mathbb{N}}\right.$ is definable in $\left(\mathbb{F}_{p}[t],+, A\right)$.

Claim 7.9. $t^{\mathbb{N}}$ and $B:=\left\{t^{i+j}-t^{i}-t^{j}: i, j \in \mathbb{N} \backslash\{0\}\right\}$ are both definable in $\left(\mathbb{F}_{p}[t],+, A\right)$.

Proof. We first check $t^{\mathbb{N}}$. I claim that if $\varphi(x)$ is $(x \in A) \wedge(2 x \in A)$ then $\varphi$ defines $t^{\mathbb{N}}$. It is clear that $\varphi\left(\mathbb{F}_{p}[t]\right) \supseteq t^{\mathbb{N}}$; suppose conversely that $f \in \varphi\left(\mathbb{F}_{p}[t]\right)$. If we had $f=\overline{2} t^{i} \in \overline{2} t^{\mathbb{N}}$ then $2 f=\overline{4} t^{i}$, and hence $2 f \notin A$ (since $p \geq 7$ implies $\overline{4} \notin\{\overline{1}, \overline{2}, \overline{3}\}$, and these are the only leading coefficients of elements of $A$ ). But this contradicts the assumption that $f \in \varphi\left(\mathbb{F}_{p}[t]\right)$. Similarly if we had $f=\overline{3} t^{i+j}-\overline{3} t^{i}-\overline{3} t^{j}$ for some $i, j$ then $2 f=\overline{6} t^{i+j}-\overline{6} t^{i}-\overline{6} t^{j}$ has leading coefficient $\overline{6}$; so by similar reasoning we conclude that $2 f \notin A$, a contradiction. So having eliminated the other possibilites we conclude that $f \in t^{\mathbb{N}}$, as desired. Note then that $\overline{2} t^{\mathbb{N}}=2 t^{\mathbb{N}}$ is also definable.

We now check $B$. I claim that if $\psi(x)$ is $3 x \in A \backslash\left(t^{\mathbb{N}} \cup 2 t^{\mathbb{N}}\right)$ then $\psi$ defines $B$. That $\psi\left(\mathbb{F}_{p}[t]\right) \subseteq B$ is just injectivity of $f \mapsto 3 f$; that $\psi\left(\mathbb{F}_{p}[t]\right) \supseteq B$ is that $\left\{\overline{3} t^{i+j}-\overline{3} t^{i}-\overline{3} t^{j}: i, j \in \mathbb{N}\right\} \cap\left(t^{\mathbb{N}} \cup \overline{2} t^{\mathbb{N}}\right)=\emptyset$, which follows by considering the leading coefficients.

So it suffices to show that $\times\left\lceil t^{\mathbb{N}}\right.$ is definable in $\left(\mathbb{F}_{p}[t],+, t^{\mathbb{N}}, B\right)$. In fact if $\varphi(x, y, z)$ is

$$
\left(x=\overline{1} \wedge z=y \in t^{\mathbb{N}}\right) \vee\left(y=\overline{1} \wedge z=x \in t^{\mathbb{N}}\right) \vee\left(x, y, z \in t^{\mathbb{N}} \wedge z-x-y \in B\right)
$$

then $\times\left\lceil t^{\mathbb{N}}=\varphi\left(\mathbb{F}_{p}[t]\right)\right.$. It is clear that $\times\left\lceil t^{\mathbb{N}} \subseteq \varphi\left(\mathbb{F}_{p}[t]\right)\right.$; for the converse suppose $\left(\begin{array}{c}t^{i} \\ t^{j} \\ t^{k}\end{array}\right) \in \varphi\left(\mathbb{F}_{p}[t]\right)$. Note that if either of the first two disjuncts of $\varphi$ holds then $\left(\begin{array}{c}t^{i} \\ t^{j} \\ t^{k}\end{array}\right) \in \times\left\lceil t^{\mathbb{N}}\right.$; suppose then that the last holds. So there are $i^{\prime}, j^{\prime}>0$ such that $t^{k}-t^{i}-t^{j}=t^{i^{\prime}+j^{\prime}}-t^{i^{\prime}}-t^{j^{\prime}}$. Since $i^{\prime}, j^{\prime}>0$ we get that the leading coefficient of the right-hand side is 1 ; for the same to be true on the left-hand side we must have $k>i, j$, and thus $k=i^{\prime}+j^{\prime}$. So $t^{i}+t^{j}=t^{i^{\prime}}+t^{j^{\prime}}$, and $\{i, j\}=\left\{i^{\prime}, j^{\prime}\right\}$. Thus $i+j=i^{\prime}+j^{\prime}=k$, and $\left(\begin{array}{c}t^{i} \\ t^{j} \\ t^{k}\end{array}\right) \in \times i t^{\mathbb{N}} . \quad \square$ Theorem 7.8

\section{References}

[1] Jason Bell, Dragos Ghioca, and Rahim Moosa. "Effective isotrivial Mordell-Lang in positive characteristic". In: arXiv preprint arXiv:2010.08579 (2020) (cit. on pp. 1, 2).

[2] Jason Bell and Rahim Moosa. "F-sets and finite automata". In: Journal de théorie des nombres de Bordeaux 31.1 (2019), pp. 101-130 (cit. on pp. 1-6, 8-12, 14, 16, 20, 21).

[3] Véronique Bruyere et al. "Logic and p-recognizable sets of integers." In: Bulletin of the Belgian Mathematical Society Simon Stevin (1994) (cit. on p. 20).

[4] Gabriel Conant and Michael C. Laskowski. "Weakly minimal groups with a new predicate". In: Journal of Mathematical Logic (2020). ISSN: 0219-0613 (cit. on pp. 19, 21, 22). 
[5] Christopher D. C. Hawthorne. "Automata and tame expansions of $(\mathrm{Z},+)$ ". In: arXiv preprint arXiv:2007.00070 (2020) (cit. on pp. 10, 15-19).

[6] Rahim Moosa and Thomas Scanlon. "F-structures and integral points on semiabelian varieties over finite fields". In: American Journal of Mathematics 126.3 (2004), pp. 473-522 (cit. on pp. 1, 2, 15-18).

[7] Sheng Yu. "Regular Languages". In: Handbook of Formal Languages: Volume 1 Word, Language, Grammar. Ed. by Grzegorz Rozenberg and Arto Salomaa. Berlin, Heidelberg: Springer Berlin Heidelberg, 1997, pp. 41-110. ISBN: 978-3-642-59136-5. DOI: 10.1007/978-3-642-59136-5_2 (cit. on pp. 2, 3, 6, 11, $12)$. 